ARTICLE

\title{
A novel IncRNA Discn fine-tunes replication protein A (RPA) availability to promote genomic stability
}

Lin Wang (1) 1,2,7, Jingzheng $\mathrm{Li}^{1,2,3,7}$, Hu Zhou (10 4, Weidao Zhang ${ }^{1,2}$, Jing Gao ${ }^{4}$ \& Ping Zheng (1) 1,2,5,6凶

RPA is a master regulator of DNA metabolism and RPA availability acts as a rate-limiting factor. While numerous studies focused on the post-translational regulations of RPA for its functions, little is known regarding how RPA availability is controlled. Here we identify a novel IncRNA Discn as the guardian of RPA availability in stem cells. Discn is induced upon genotoxic stress and binds to neucleolin (NCL) in the nucleolus. This prevents NCL from translocation into nucleoplasm and avoids undesirable NCL-mediated RPA sequestration. Thus, Discn-NCL-RPA pathway preserves a sufficient RPA pool for DNA replication stress response and repair. Discn loss causes massive genome instability in mouse embryonic stem cells and neural stem/progenigor cells. Mice depleted of Discn display newborn death and brain dysfunctions due to DNA damage accumulation and associated inflammatory reactions. Our findings uncover a key regulator of DNA metabolism and provide new clue to understand the chemoresistance in cancer treatment.

\footnotetext{
${ }^{1}$ State Key Laboratory of Genetic Resources and Evolution, Kunming Institute of Zoology, Chinese Academy of Sciences, Kunming, Yunnan 650223, China. ${ }^{2}$ Yunnan Key Laboratory of Animal Reproduction, Kunming Institute of Zoology, Chinese Academy of Sciences, Kunming, Yunnan 650223, China.

${ }^{3}$ University of Chinese Academy of Sciences, Beijing 101408, China. ${ }^{4}$ Department of Analytical Chemistry and CAS Key Laboratory of Receptor Research, Shanghai Institute of Materia Medica, Chinese Academy of Sciences, Shanghai 201203, China. ${ }^{5}$ KIZ/CUHK Joint Laboratory of Bioresources and Molecular Research in Common Diseases, Kunming Institute of Zoology, Chinese Academy of Sciences, Kunming, Yunnan 650223, China. ${ }^{6}$ Center for Excellence in Animal Evolution and Genetics, Chinese Academy of Sciences, Kunming 650223, China. ${ }^{7}$ These authors contributed equally: Lin Wang, Jingzheng Li.

$\varpi_{\text {email: zhengp@mail.kiz.ac.cn }}$
} 
S tem cells (SCs, including pluripotent embryonic stem cells and tissue stem cells) are cellular basis for organism development and tissue homeostasis. Genetic lesions in SCs usually induce the loss of stem cell identity, causing numerical or functional perturbations in SCs. These perturbations can have catastrophic consequences for tissue and organismal homeostasis, resulting in embryonic lethality, developmental defects, degenerative disorders, and oncogenesis ${ }^{1}$. In adaptation to their functional significance, SCs are able to maintain superior stable genome. The genome maintenance ability in SCs lies in their unique DNA metabolic properties in aspects of DNA replication and repair. Limited pioneer works showed that mouse embryonic stem cells (mESCs) utilize ZSCAN4-mediated DNA recombination-based pathway to lengthen the telomere ${ }^{2}$. ESCs do not use TRF2 to protect the telomere, whereas TRF2 is essential for telomere protection in somatic cells ${ }^{3,4}$. Compared to somatic cells, ESCs have superior abilities to resolve replication stress and repair DNA damages. Several stem cell-specific proteins, including SALL4, FILIA, and FILIA-FLOPED protein complex were identified as the underlying regulators ${ }^{5-7}$. Despite these progresses, how SCs efficiently replicate and repair DNA during frequent cell proliferation still remains largely unclear. Comprehensive elucidation of the underpinning mechanisms would largely expand our knowledge on the regulations of DNA metabolism and genomic stability maintenance in stem cells, and help understand the relevant pathophysiological conditions ${ }^{1}$.

During DNA metabolism including DNA replication, repair and recombination, single-stranded DNA (ssDNA) is frequently generated and rapidly coated by replication protein A (RPA) complex. RPA is composed of three subunits RPA70, RPA32, and RPA14, and is the major ssDNA-binding protein in eukaryotic cells. RPA binding to ssDNA not only protects the naked ssDNA from nucleolytic degradation and prevents the secondary struction formation in ssDNA, but also serves as a platform to launch downstream events in all DNA metabolic processes. Thus, RPA acts as a master regulator in DNA metabolism. RPA perturbations, for instance RPA haploinsufficiency, depletion, or exhaustion, can result in DNA replication catastrophe, DNA repair defects and genomic instability. Given its central roles, RPA has emerged as a focus for cellular responses to genotoxic stress ${ }^{8-10}$. To date, the majority of studies investigated the regulations of RPA functions in different aspects of DNA metabolism, and revealed the post-translational modifications including phosphorylation, SUMOylation, and ubiquitination as important regulators ${ }^{10}$. Compared to post-translational modifications, however, little is known on how cells regulate RPA availability, which is a rate-limiting factor in RPA functions.

DNA replication stress and DNA DSBs can generate massive ssDNA, which rapidly exhausts RPA reservoir. Increasing RPA availability is essential to improve cellular resistence to these stresses. Compared to somatic cells, ESCs are more tolerant to DNA DSBs and replication stress ${ }^{7,11}$. This raised a hypothesis that ESCs may develop unique strategies to better sustain free RPA pool. In this study, we aimed to understand the mechanisms regulating the resistence of ESCs to DNA replication stress and DNA DSBs. We focused on long noncoding RNAs (lncRNAs), which can modulate and fine-tune gene expression and protein functions through interacting with DNA, RNAs, and proteins in many cellular events ${ }^{12}$. By comparing the RNA expression profiles of mouse ESCs and their differentiated progenies under unperturbed and genotoxic treatment conditions, we identified a short list of lncRNAs that were specifically induced in ESCs upon both replication stress and DNA DSBs. We then characterized in detail an un-annotated lncRNA Discn (DNA damage-induced stem cell specific noncoding RNA), whose expression displayed the most robust increase in response to DNA replication stress and DSBs. Our results showed that Discn ensured sufficient RPA availability via Discn-NCL-RPA pathway to promote genome stability. Notably, knockout of Discn in mice caused newborn death as well as brain dysfunctions, demonstrating its important physiological functions in normal embryonic development.

\section{Results}

A novel lncRNA Discn responds to genotoxic stress in stem cells. To explore if there exist unique $\ln \mathrm{R} N A s$ responsive to DNA replication stress and DNA DSBs in stem cells, we performed RNA-seq and compared the genome-wide RNA expression profiles of mouse ESCs to their differentiated progenies cultured under unperturbed or genotoxic conditions (hydroxyurea or etoposide treatment $)^{13}$. The cellular identity of differentiated progenies was validated by the expression levels of pluripotency genes and three germ layer markers (Supplementary Fig. 1a). We identified a short list of lncRNAs (Supplementary Fig. 1b), which were predominantly expressed in ESCs and significantly stimulated by genotoxic treatments. Among them, an un-annotated lncRNA displayed the most robust response to genotoxic stress, with expression level increasing more than twenty fold after hydroxyurea, etoposide, mitomycin C, or camptothecin treatment (Fig. 1a). We, therefore, named this lncRNA as Discn (DNA damage-induced stem cell specific noncoding RNA). We also treated ESCs with hydroxyurea plus ATR inhibitor VE-821, which can robustly enhance the unscheduled origin firing and ssDNA formation under replication stress. Intriguingly, inhibition of ATR signaling during replication stress further stimulated the expression of Discn in a dose-dependent manner (Fig. 1b). This expression pattern suggested that Discn was responsive to the generation of ssDNA, and might be involved in regulation of genomic stability via ssDNA-related events.

To better understand Discn, we obtained its full length sequence (GenBank accession number: MZ269527) using 3'and $5^{\prime}$-end RACE (rapid amplification of cDNA ends) (Supplementary Fig. 1c). Discn is located on chromosome 11 and contains two exons (Supplementary Fig. 1d). Although several potential coding frames are present in the sequence (Supplementary Fig. 1c), they did not translate into proteins or peptides (Supplementary Fig. 1e). Thus, Discn is a potential noncoding RNA. We also observed the expression of Discn in several available mouse tissue stem/progenitor cell samples (e.g., cultured mouse spermatogonia stem cells, mouse neural stem/ progenitor cells (NSPCs), mammary stem cells) (Supplementary Fig. 1f). However, Discn was not detected in many organs except of brain and testis (Supplementary Fig. 1g). In addition, Discn is conserved among mammalian species (Supplementary Fig. 1h). These data collectively proposed a potential and probably a general role of Discn in regulating stem cell genomic stability.

Discn safeguards genomic stability of cultured stem cells. To find out if Discn is required to maintain genomic stability of stem cells, we first evaluated its functions in in-vitro cultured stem cells. Discn was efficiently knocked down (KD) via two independent short hairpin RNAs (shRNAs) in mESCs (Supplementary Fig. 1i). Under unperturbed culture condition, Discn KD mESCs displayed elevated level of DNA DSBs, as monitored by increased amount of $\gamma \mathrm{H} 2 \mathrm{AX}$ (Fig. 1c). The severe DNA DSBs were further validated by neutral comet assay (Fig. 1d), which measures the DSBs at single cell resolution ${ }^{14}$. In addition, many chromosome instability (CIN) phenotypes including aneuploidy (Fig. 1e), chromosome translocation measured by sister chromatid exchange (SCE) (Fig. 1f), and micronuclei formation (Fig. 1g), were prevalent in Discn KD mESCs. However, the 

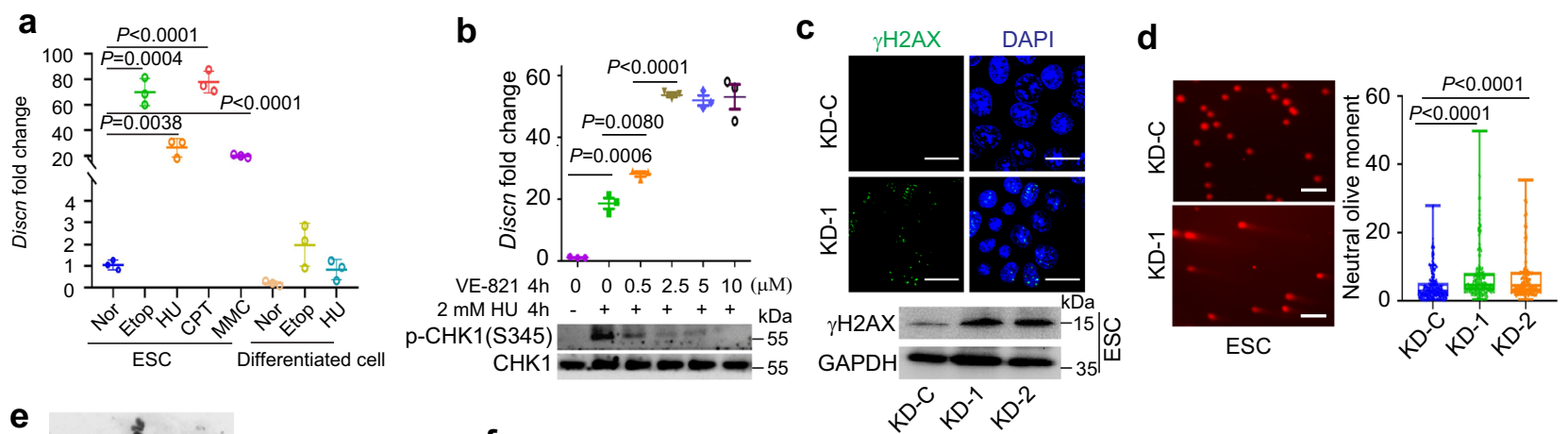

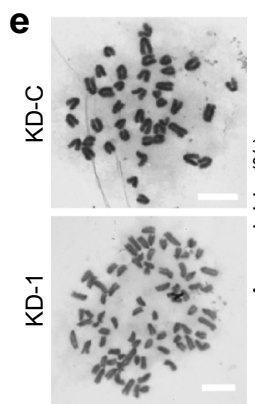

ESC at P35

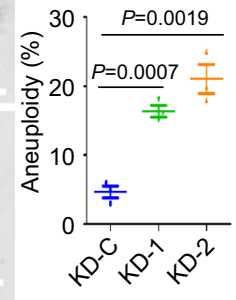

ESC at P35 f

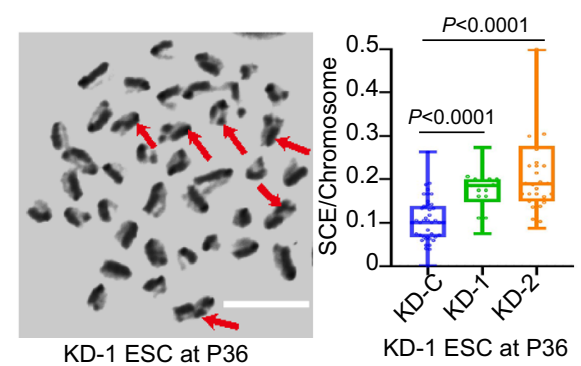

g

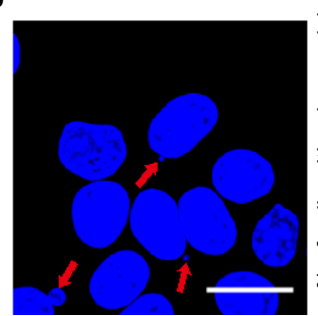

KD-1 ESC at P50

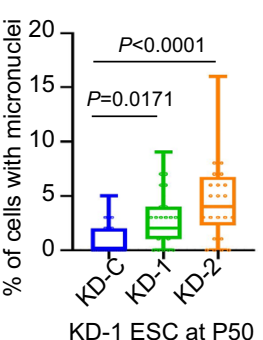

Fig. 1 Discn safeguards genomic stability of mouse ESCs. a Mouse ESCs and their differentiated isogenic cells were cultured in normal condition (Nor) or treated with $2 \mathrm{mM}$ hydroxyurea (HU), $10 \mu \mathrm{M}$ etoposide (Etop), $1 \mu \mathrm{M}$ camptothecin (CPT), or $8 \mu \mathrm{g} / \mathrm{mL}$ mitomycin $\mathrm{C}$ (MMC) for $4 \mathrm{~h}$. The relative expression level of Discn was determined by quantitative RT-PCR. The amount in ESCs under normal condition was set as 1.0 . $n=3$ biologically independent samples. Data were presented as mean \pm SEM, two-tailed unpaired $t$ test. b Quantitative RT-PCR showed Discn expression was increased by HU-induced replication stress. Blockage of ATR signaling by inhibitor VE-821 further stimulated the expression of Discn in a dose-dependent manner. The Discn amount in ESCs under normal condition was set as 1.0. Upper panel showed the Discn expression fold changes and lower panel was the validation of ATR inhibition by VE821. $n=3$ biologically independent samples, two-tailed unpaired $t$ test. Data were presented as mean \pm SEM. c Compared to control (KD-C), knockdown of Discn by two independent shRNAs (KD-1 and KD-2, respectively) increased DNA double-strand breaks (DSBs) as monitored by the increased level of $\gamma \mathrm{H} 2 \mathrm{AX}$. Upper panel showed the representative immunostaining images. Lower panel showed the immunoblotting result. Three independent experiments were repeated with similar results. Scale bar, $20 \mu \mathrm{m}$. d Neutral comet assay validated the increased DNA DSBs in Discn KD cells. Left panel, the representative images. Right panel, the quantification of comet tail length. At least 100 tails were analyzed in each group in three independent replications. Scale bar, $400 \mu \mathrm{m}$. e Discn KD ESCs had higher rate of aneuploidy. Representative image at passage 35 (P35) (Left panel) and the quantification (right panel) were obtained from at least 50 metaphase spreads in three independent replications. Data were shown as mean \pm SEM, two-tailed Student's $t$-test. Scale bar, $10 \mu \mathrm{m}$. f Discn KD increased the sister chromatid exchange rates. Left panel showed the representative images of sister chromatid exchange (arrows) in KD ESCs at passage 36. Quantification was shown on the right. $n=3$ biologically independent samples, at least 20 metaphase spreads were analyzed in each group. Scale bar, $10 \mu \mathrm{m}$. $\mathbf{g}$ Discn KD increased micronuclei formation. Left panel showed the representative images of micronuclei

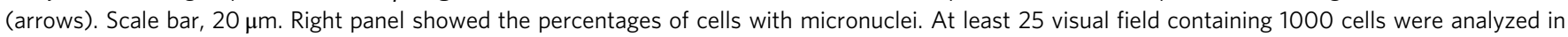
three replications. $\mathbf{d}, \mathbf{f}, \mathbf{g}$ Data were representative of individual values with box and whiskers plots showing the median, upper and lower quartiles, and minimum and maximum. Two-tailed Student's t-test.

mRNA expressions of core pluripotency regulators Nanog, Klf4, Sox2, and Oct4 were not affected (Supplementary Fig. 1j). Consistently, the mutant mESCs displayed normal morphology (Supplementary Fig. 1k), although their proliferation rates were reduced (Supplementary Fig. 11).

We also examined the role of Discn in cultured mouse NSPCs. After knocking down Discn (Supplementary Fig. 2a), we consistently observed the severe genome instability in NSPCs (Supplementary Fig. 2b-e). Thus, these results collectively demonstrated a general and critical function of Discn in ensuring genome stability of in-vitro propagated stem cells.

Discn regulates replication stress response and HR-mediated DNA repair. We next went on to elucidate the functions and mechanisms of Discn using mouse ESC as a model. Endogenous DNA DSBs frequently arise when the DNA replication and/or repair are compromised. In response to replication stress, cells elicit coordinated reactions, which locally protect and repair the stalled forks, and globally inhibit cell cycle progression and dormant origin firing in order to avoid excessive ssDNA generation $^{15}$. To find out if Discn depletion compromises DNA replication stress response, we induced fork stalling by hydroxyurea treatment and examined the local and global responses of cells. ATR-CHK1 signaling plays a central coordination role in replication stress response. In WT ESCs, ATR kinase was robustly activated and efficiently sustained, as monitored by the persistent phosphorylation of its downstream effector CHK1 at Ser $345^{16}$. However, ATR signaling failed to be well maintained when Discn was lost, although its initial activation was grossly normal (Fig. 2a). We then utilized DNA fiber assay to closely examine the behaviors of replication forks ${ }^{17}$. Compared to Discn proficient cells, the stalled forks in Discn KD cells were prone to degradation under hydroxyurea treatment (Fig. 2b) and difficult to restart after release from stress (Fig. 2c). This indicated that the local responses to protect/repair stalled forks were compromised when Discn was absent. Notably, more dormant replication origins were fired (Fig. 2d) and significantly higher level of ssDNA was detected in Discn KD ESCs following hydroxyurea treatment (Fig. 2e), demonstrating that the global response to suppress unscheduled origin firing was also impaired by Discn KD. As a result, Discn KD ESCs were less tolerant to replication stress and 

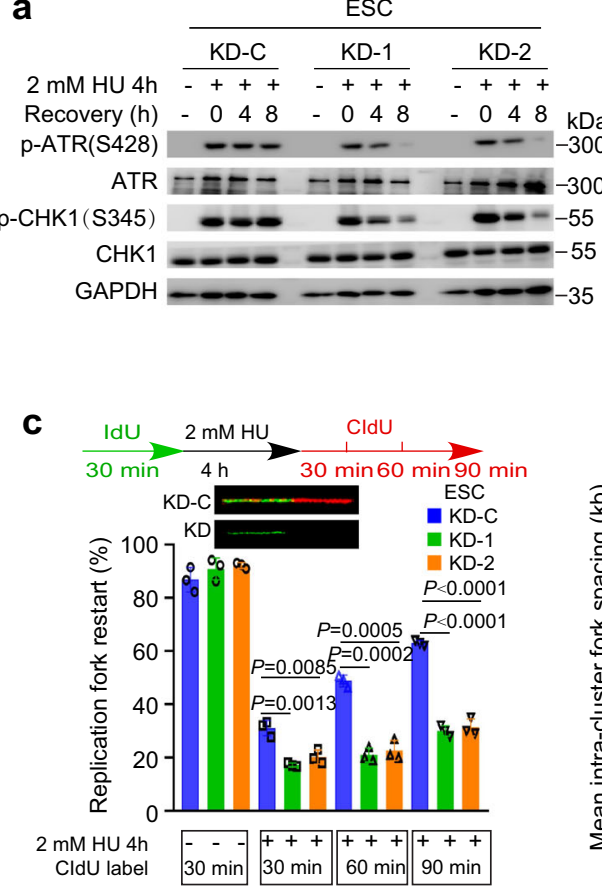

C

ESC $\quad$ g

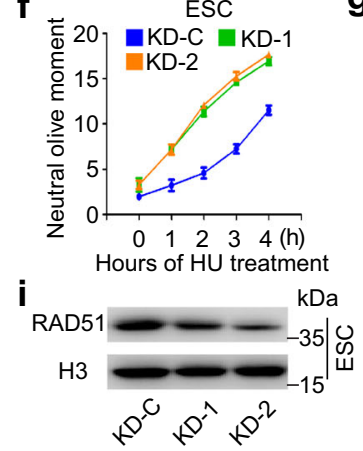

g

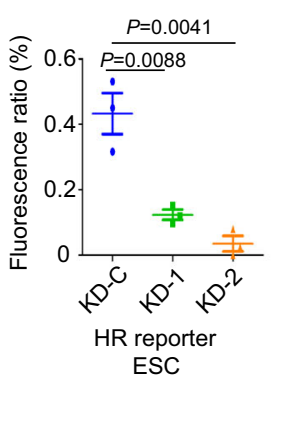

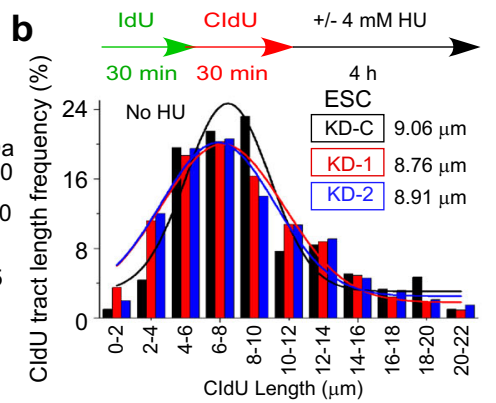

CldU Length $\check{(} \mu \mathrm{m})$

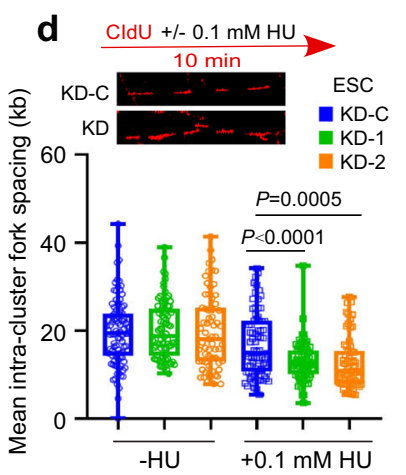

e
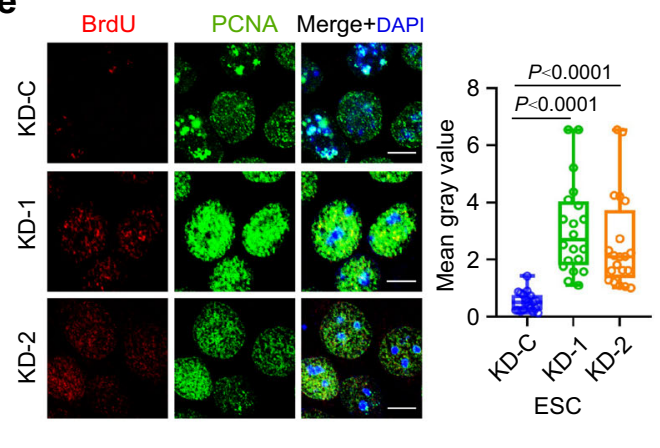

Fig. 2 Discn regulates DNA replication stress response and HR-mediated DNA DSB repair. a Mouse ESCs were treated with hydroxyurea (HU) to induce replication stress. ATR-CHK1 kinase activation failed to be well sustained in two Discn knockdown cells (KD-1 and KD-2) when compared to the knockdown control (KD-C). Three independent experiments were repeated with similar results. b DNA fiber assay revealed that the stalled forks in Discn KD cells were prone to undergo degradation after HU treatment. 200 fibers from three independent replications were analyzed. c Discn KD compromised the stalled fork restart. At least 200 fibers from three independent replications were analyzed. d Discn KD enhanced dormant origin firing as indicated by the reduced mean fork spacing. At least 50 continuous fibers from three independent replications were analyzed. e Discn KD increased the content of ssDNA as measured by the native BrdU incorporation assay at S phase. Scale bar, $10 \mu \mathrm{m}$. Quantification is shown on the right. At least 20 images were analyzed in each condition. $\mathbf{f}$ Compared to control ESCs (KD-C), Discn KD cells were more sensitive to hydroxyurea treatment and accumulated more DNA DSBs as measured by neutral comet assay. Data were from three replications and shown as mean \pm SEM. $\mathbf{g}$ FACS analysis of reporter ESCs showed that Discn KD suppressed HR-mediated DNA DSB repair, but inversely stimulated NHEJ repair pathway. Data were from three independent experiments. $\mathbf{h}$ DNA DSBs were generated by laser microirradiation. Discn KD impaired the recruitment of Rad51 to DSB sites labeled with $\gamma \mathrm{H} 2 \mathrm{AX}$ at $\mathrm{S}$ phase ( $\mathrm{BrdU}+\mathrm{H}^{+}$), indicating the suppression of HR pathway. Right panel showed the proportions of S phase cells capable of HR repair (three replicates, 50 cells in each replicate). Scale bar, $5 \mu \mathrm{m}$. i Immunoblotting showed a decrease in chromatin-bound Rad51 in Discn KD ESCs synchronized at S phase. Three independent experiments were repeated with similar results. $\mathbf{b}, \mathbf{c}, \mathbf{f}-\mathbf{h}$ Data were shown as mean \pm SEM, two-tailed Student's $t$-test. $\mathbf{d}$, e Data were representative of individual values with box and whiskers plots showing the median, upper and lower quartiles, and minimum and maximum. Two-tailed Student's $t$-test.

produced more DSBs at different time-points of hydroxyurea treatment when compared to Discn proficient ESCs (Fig. 2f).

Because Discn expression was also induced by etoposide treatment which generates DNA DSBs ${ }^{13}$, we examined its possible involvement in DSB repair. DNA DSBs are repaired by two major pathways: homologous recombination (HR) and classical nonhomologous end-joining (NHEJ) pathways, both of which can be monitored through engineered GFP-based reporters ${ }^{18}$. In the reporter cells, site-specific DSBs are generated in a cassette after expression of endonuclease I-SceI. Repair of the
DSBs by respective HR or NHEJ pathway restores the functional RFP or GFP gene and the numbers of RFP- or GFP-expressing cells counted by flow cytometry provide quantitative measure of HR or NHEJ efficiency. To this end, we efficiently knocked down Discn in these reporter mESC lines by using the aforementioned shRNAs, and measured the respective $\mathrm{HR}$ and NHEJ repair efficiency. In Discn KD cells, HR repair was suppressed, whereas NHEJ pathway was inversely stimulated probably due to repair compensation (Fig. 2g). To further validate the impairment of $\mathrm{HR}$ pathway, we examined the recruitment of recombinase RAD51 to 
DSB sites, which is essential for HR repair ${ }^{19}$. Following laser micro-irradiation, much less Discn KD cells recruited RAD51 to DSB sites (labeled with $\gamma \mathrm{H} 2 \mathrm{AX}$ ) at S-phase of cell cycle when compared to WT ESCs (Fig. 2h). In concordance, the amounts of chromatin-bound RAD51 proteins were significantly reduced by Discn KD in ESCs synchronized at $\mathrm{S}$ phase and treated with etoposide (Fig. 2i). Taken together, these data support that Discn regulates DNA replication stress response and HR-mediated DNA DSB repair.

Discn accumulates in nucleolus and binds to nucleolin (NCL). To understand how Discn regulates replication stress response and HR-mediated DNA repair, we first investigated its subcellular localization under normal and stressed conditions by RNA fluorescence in situ hybridization (RNA-FISH). No specific distribution pattern of Discn was detected when using conventional staining procedures. However, following extraction of soluble Discn with Triton X-100, we observed faint staining of Discn in nucleolus labeled with fibrillarin (FBL) under normal culture condition. Hydroxyurea treatment significantly stimulated the accumulation of Discn in nucleolus (Fig. 3a). Fractionation of nucleolus (Supplementary Fig. 3a) followed by quantitative RT-PCR examination verified the increased amount of Discn in nucleolus after hydroxyurea treatment (Fig. 3b). Of note, Discn exhibited the same subcellular localization in response to etoposide treatment (Fig. 3a, b), suggesting that a common mechanism underlies the dual regulations of Discn on replication stress response and HR-mediated DNA repair.

Next, we performed in vitro RNA pulldown followed by mass spectrometry analysis to identify potential interaction proteins of Discn (Supplementary Fig. 3b). Among the list of candidates, a nucleolus protein nucleolin (NCL) showed relatively high score (Supplementary Fig. 3c). Immunoblotting examination of NCL on in vitro RNA pulldown samples validated the association of Discn with NCL (Supplementary Fig. 3d). Because in vitro binding can yield false-positive interaction, we performed in vivo cross-linking followed by RNA pulldown to validate their interaction. Notably, while weak interaction was detected in nuclear fraction under unperturbed condition, strong association was observed after hydroxyurea or etoposide treatment (Fig. 3c). To further confirm the association of Discn with NCL, we conducted RNA immunoprecipitation to examine whether Discn can be co-immunoprecipitated with NCL under native condition. As shown, very few Discn RNAs were detected in immunoprecipitated samples in unperturbed condition. However, genotoxic treatments robustly increased the amount of Discn associated with NCL (Fig. 3d, e). In line with their association, immunostaining showed the co-localization of Discn with NCL in nucleolus upon genotoxic stress (Fig. 3f). Collectively, these data support that Discn binds to NCL in nucleolus and their association is enhanced by genotoxic stress.

We went on to map the NCL binding sites on Discn. NCL has binding motif in target RNAs. The motif is G-rich and has stemloop secondary structures. NCL is able to associate with RNAs bearing this motif in the absence of other proteins ${ }^{20}$. Using RegRNA 2.0 online tools (http://regrna2.mbc.nctu.edu.tw/ index.html) ${ }^{21}$, we identified several putative NCL binding sites predominantly at the $5^{\prime}$ end of Discn. We then constructed the Discn mutants containing either the $5^{\prime}$ end (2-156 bp), $3^{\prime}$ end (625-823 bp), or central part (157-630 bp). In vitro RNA pull down assay revealed that the NCL binding sites were predominantly localized at the $5^{\prime}$ end (Fig. $3 \mathrm{~g}$ ).

Discn-NCL interaction does not require genotoxic stimuli. Genotoxic stress stimulated the expression of Discn as well as its association with NCL. To elucidate whether the enhanced Discn-
NCL interaction was simply due to the increased Discn expression or required genotoxic stimuli, we stably expressed Discn in WT mESCs (Supplementary Fig. 3e) and re-examined the Discn-NCL association. Intriguingly, under unperturbed culture conditions Discn localized in nucleolus (Supplementary Fig. 3f) and associated with NCL as detected by in vivo RNA pulldown (Supplementary Fig. 3g). Notably, genotoxic treatment did not further increase their association (Supplementary Fig. 3g).

To gain more evidence, we ectopically expressed Discn in mouse embryonic fibroblasts (MEFs) (Supplementary Fig. 3h). Concordantly, Discn appeared in nucleolus of MEFs (Fig. 3h) and associated with NCL as detected by in vivo RNA pulldown assay (Fig. 3i). Of note, the association was not affected by genotoxic treatments (Fig. 3i). Thus, localization of Discn in nucleolus as well as Discn-NCL interaction does not require the genotoxic signaling and rather it seems cell autonomous.

Discn sequesters NCL in nucleolus to preserve the free RPA pool. NCL predominantly resides in nucleolus under unperturbed conditions. However, two studies on somatic cells reported that upon heat shock or genotoxic stress, part of the nucleolar NCL pool translocates into the nucleoplasm, where it binds to $\mathrm{RPA}^{22,23}$. The formation of NCL-RPA protein complex in nucleoplasm reduces the free RPA level, thereby counteracting the RPA functions.

Based on the above knowledge, we wondered whether Discn regulates genomic stability by targeting the NCL-RPA complex. To this end, we first evaluated the functional significance of Discn-NCL interaction in nucleolus. In line with previous reports, hydroxyurea or heat stress did not change NCL protein expression in MEFs. However, these treatments drastically increased the relocation NCL into nucleoplasm, and conversely decreased the retention of NCL in nucleolus, as determined by the fractionation of nucleolus and nucleoplasm combined with immunoblotting analysis (Fig. 4a). Intriguingly, we observed a distinctive pattern of NCL distribution in mESCs, in which the NCL protein level in nucleoplasm remained comparable before and after genotoxic treatments (Fig. 4b). Concordantly, NCL retention in nucleolus was not obviously affected by genotoxic stress (Fig. 4b). Thus, unlike in differentiated cells, genotoxic stress does not evoke obvious NCL translocation from nucleolus to nucleoplasm in mESCs.

We then examined if Discn loss influenced NCL distribution in mESCs. Unlike in Discn proficient ESCs, genotoxic treatment caused part of the nucleolar NCL pool relocation to nucleoplasm in Discn KD ESCs, as manifested by a substantial increase of NCL level in nucleoplasm. Due to the high abundance of NCL in nucleolus, release of part of the nucleolar NCL pool into nucleoplasm caused a mild but visible reduction of NCL level in nucleolus (Fig. 4b). The translocation of NCL from nucleolus to nucleoplasm in Discn KD ESCs was further verified by immunofluorescence analysis (Supplementary Fig. 3i). Concordantly, ectopic expression of Discn in MEFs significantly attenuated the translocation of NCL from nucleolus to nucleoplasm (Fig. 4c). We also expressed the three Discn fragments (5 end, $3^{\prime}$ end, and central part), respectively, in MEFs and examined their influence on NCL translocation. In line with their ability to bind to NCL (Fig. 3g), 5' end fragment displayed the higher efficiency to prevent NCL translocation to nucleoplasm than $3^{\prime}$ end, whereas central part had no effect (Fig. 4d). These data altogether supported that Discn-NCL interaction sequestered NCL in nucleolus.

In somatic cells, NCL forms protein complex with RPA in nucleoplasm ${ }^{23}$. Given that the NCL protein level increased drastically in nucleoplasm of Discn KD ESCs (Fig. 4b), we speculated that NCLRPA association was subsequently enhanced. Indeed, co- 


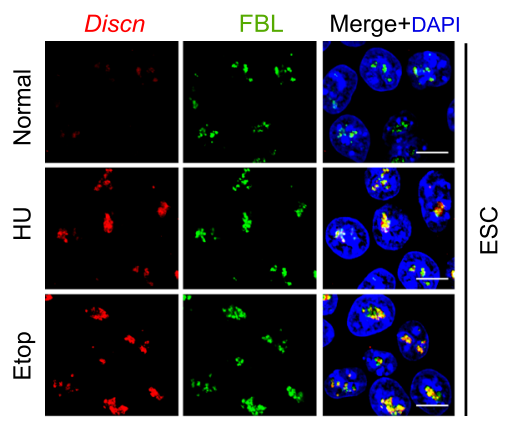

b

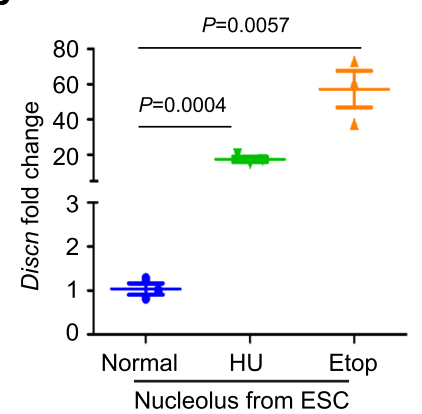

C

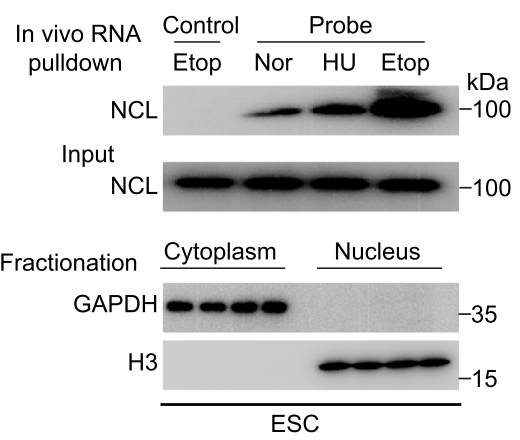

d

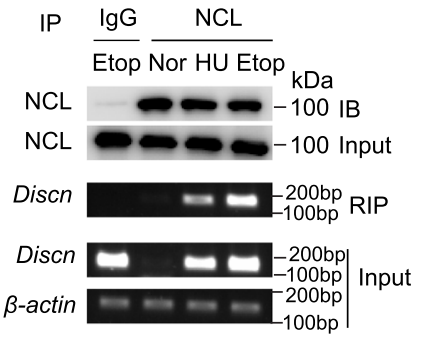

g
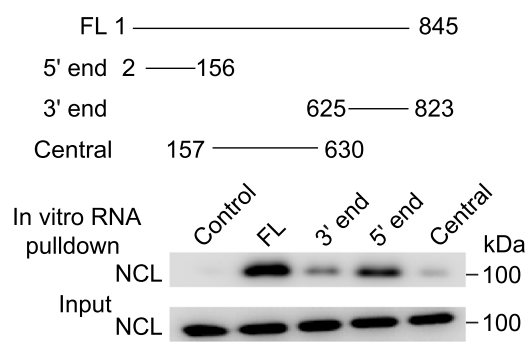

e

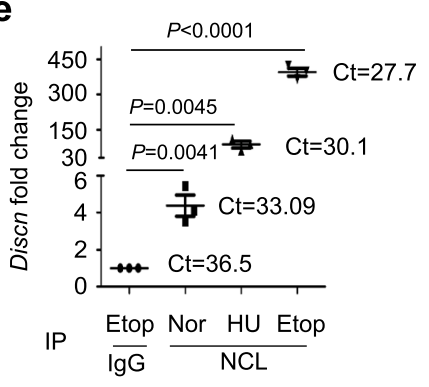

f

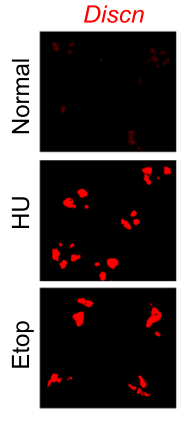

FBL

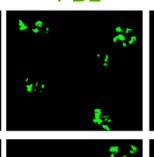

NCL

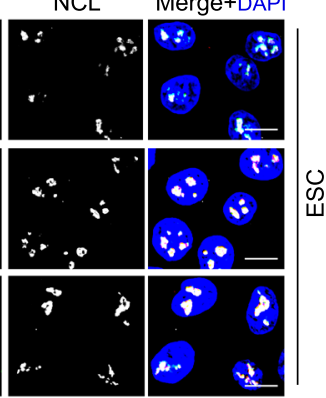

h

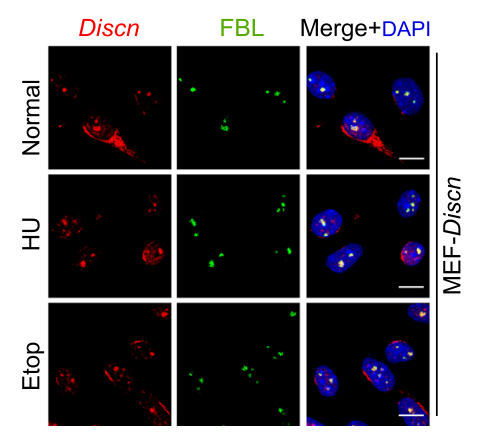

i

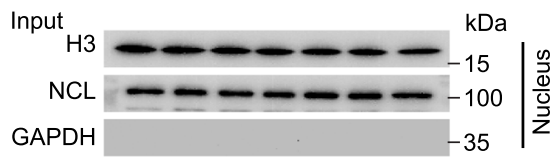

In vivo RNA pulldown

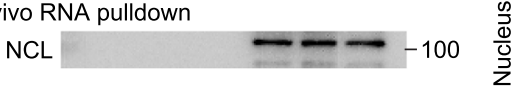

Etop Nor HU Etop Nor HU Etop

MEF-Discn
Control

Fig. 3 Discn localizes in nucleolus and interacts with nucleolin (NCL). a mESCs were cultured under normal condition or treated with $2 \mathrm{mM}$ hydroxyurea $(\mathrm{HU})$ or $10 \mu \mathrm{M}$ etoposide (Etop) for $4 \mathrm{~h}$. RNA-FISH detected the localization of Discn in nucleolus labeled with fibrillarin (FBL) upon treatments. Three independent experiments were repeated with similar results. $\mathbf{b}$ Nucleolus was fractionated and quantitative RT-PCR showed the increased accumulation of Discn in nucleolus after HU or etoposide treatment. rRNA was used as control to calculate the relative expression of Discn. The amount of Discn in nucleolus of ESCs under normal condition was set as 1.0. Data were shown as mean \pm SEM from three replications. Two-tailed Student's $t$-test. c ESCs were fractionated into compartments of cytoplasm and nucleus (lower panel). In vivo RNA pulldown combined with immunoblotting on nuclear fraction detected the Discn-NCL association under normal or genotoxic treatment conditions (upper panel). Note that treatments did not affect NCL expression (middle panel). Pulldown assay using sense probe was set as negative control. Three independent experiments were repeated with similar results. $\mathbf{d}$ RNA immunoprecipitation with NCL antibody confirmed the Discn-NCL association. Upper panel showed NCL itself was successfully precipitated. Middle panel showed the RT-PCR detection of Discn precipitated with NCL. Lower panel showed the input. Three independent experiments were repeated with similar results. e The relative levels of Discn immunoprecipitated with NCL were determined by quantitative RT-PCR. $n=3$ biologically independent samples, twotailed unpaired t test. Data were presented as mean \pm SEM. $\mathbf{f}$ Co-localization of Discn and NCL in nucleolus labeled with FBL in mESCs under normal or treatment conditions. Three independent experiments were repeated with similar results. $\mathbf{g}$ In vitro RNA pulldown assay revealed that Discn fragment containing $5^{\prime}$ end displayed high affinity to NCL. FL, full length Discn. Control, antisense Discn. Three independent experiments were repeated with similar results. $\mathbf{h}$ Co-immunostaining showed the localization of Discn in nucleolus of MEFs ectopically expressing Discn (MEF-Discn). Note that HU or etoposide treatment did not further enhance the accumulation of Discn in nucleolus. Three independent experiments were repeated with similar results. $\mathbf{i}$ MEFs with ectopic expression of Discn or transfected with vector (MEF-vector) were fractionated into cytoplasmic and nuclear compartments (upper panel). In vivo RNA pulldown using nuclear fraction validated the Discn-NCL association (lower panel). Note that compared to normal condition (Nor), HU or etoposide treatment did not further stimulate the Discn-NCL interaction. Sense probe RNA pulldown was set as negative control. Three independent experiments were repeated with similar results. Scale bar, $10 \mu \mathrm{m}$.

immunoprecipitation using antibody against RPA32, one of the RPA subunits $^{24}$, revealed a significant induction of NCL-RPA32 interaction in Discn KD mESCs compared to Discn proficient ESCs upon hydroxyurea treatment (Fig. 4e). Conversely, ectopic expression of Discn in MEFs significantly decreased the NCL-RPA32 complex formation (Fig. 4f). Because the total RPA protein expression was not influenced by Discn depletion (Supplementary Fig. 3j), we concluded that Discn KD led to the reduction of free RPA level in ESCs.

Overexpression of RPA rescues the genomic instability of Discn KD cells. Because RPA is a master regulator of DNA 

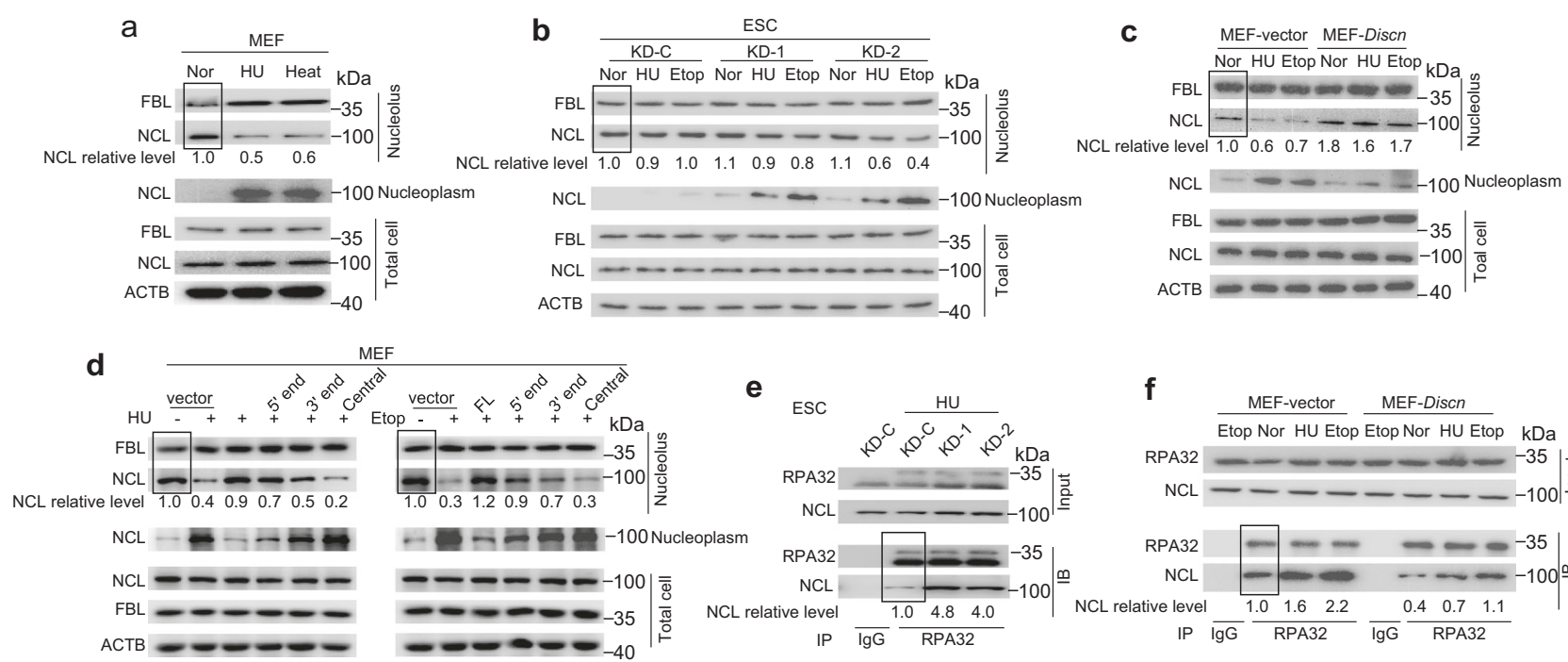

e

e ESC

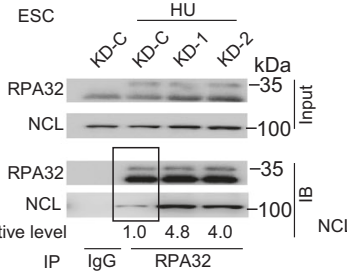

$\mathbf{f}$

MEF-vector MEF-Discn

Etop Nor HU Etop Etop Nor HU Etop $\mathrm{kDa}$
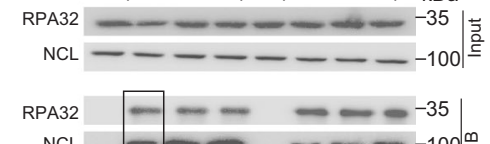

$\mathrm{NCL} \quad-\infty-100 \cong$

IP $\overline{\lg G} \frac{1.0 \quad 1.6 \quad 2.2}{\text { RPA32 }} \frac{0.4 \quad 0.7 \quad 1.1}{\lg G}$

Fig. 4 Discn sequesters NCL in nucleolus to prevent NCL-RPA association in nucleoplasm. a Nucleolus and nucleoplasm were fractionated in MEFs. Immunoblotting revealed that $\mathrm{HU}$ or heat treatment caused robust translocation of NCL from nucleolus to nucleoplasm. $\mathbf{b}$ Fractionation combined with immunoblotting showed that nucleoplasmic NCL level remained comparable before and after genotoxic treatment in Discn proficient (KD-C) mESCs. However, in Discn knockdown ESCs (KD-1, KD-2) genotoxic treatment evoked a robust increase of NCL level in nucleoplasm accompanied with a mild NCL decrease in nucleolus. c Ectopic expression of Discn in MEFs attenuated the translocation of NCL from nucleolus to nucleoplasm. d Different Discn fragments ( $5^{\prime}$ end, $3^{\prime}$ end, and central part) expressed in MEFs showed distinct efficiency to prevent the translocation of NCL from nucleolus to nucleoplasm. e Co-immunoprecipitation (Co-IP) showed that Discn KD in mESCs increased the association of NCL with RPA32. f Similarly, ectopic expression of Discn in MEFs suppressed the interaction of NCL with RPA32. Cells were cultured under normal condition (Nor) or treated with HU (2 mM, $4 \mathrm{~h}$ ), etoposide $(10 \mu \mathrm{M}, 4 \mathrm{~h})$, or heat shock $\left(44^{\circ} \mathrm{C}, 4 \mathrm{~h}\right)$. In (a-d), NCL relative levels in nucleolus were normalized by FBL. In (e, f), NCL relative levels were normalized by co-immunoprecipitated RPA32. The level in samples marked with box was set as 1 . All experiments were repeated three times with similar results.

metabolism and critical guardian of genomic integrity 25,26 , we reasoned that insufficient RPA surplus in Discn KD SCs might underpin the genomic instability defects. To test this hypothesis, we over-expressed the three subunits of RPA complex (RPA70, RPA32, and RPA14, respectively) in Discn KD ESCs (Supplementary Fig. 4a), and examined if this could rescue the defects. Notably, the cellular responses to hydroxyurea or etoposide treatment were fully restored. These included the ATR-CHK1 signaling (Fig. 5a), the stalled fork stabilization (Fig. 5b) and restart (Fig. 5c), the suppression of unscheduled origin firing (Fig. 5d) and ssDNA formation (Fig. 5e), the tolerance to hydroxyurea-induced replication fork breakage (Fig. 5f), and the HR-mediated DSB repair (Fig. 5g). Concordantly, the overall DNA DSB contents (Supplementary Fig. 4b) and the cell proliferation rates (Supplementary Fig. 4c) of Discn KD ESCs were recovered to normal by overexpression of RPAs. We also examined if the same machanism operated in NSPCs. Similar to the observations in mESCs, overexpression of the RPA complex in cultured Discn KD NSPCs rescued the genomic instability phenotypes (Supplementary Fig. 5). Collectively, these data support that Discn-NCL-RPA pathway functions generally in SCs. Protein expression level also serves as a regulatory layer. We found that ESCs express more RPA proteins than NSPCs and MEFs, whereas NSPCs and MEFs had comparable RPA expression (Supplementary Fig. 4d). Thus, Discn adds a fine-tune regulatory layer to RPA surplus.

In conclusion, our data proposed a unique mechanism in SCs, in which lncRNA Discn is robustly induced by genotoxic stress and localizes in nucleolus where it interacts with NCL and sequesters NCL in nucleolus. Interaction of Discn-NCL in nucleolus reduces the undesirable NCL-RPA association in nucleoplasm, thereby achieving a sufficient RPA surplus crucial for DNA replication stress response and repair (Fig. 5h). In line with this model, ectopic expression of Discn in MEFs reduced the NCL-RPA association (Fig. 4f), and conferred protection against genetoxic stress (Supplementary Fig. 4e-i). However, expression of mutant Discn fragments which do not efficiently bind to NCL ( $3^{\prime}$ end and central part) failed to protect MEFs against genotoxic damage (Supplementary Fig. 4i). A recent study reported that IncRNAs, which are generally outnumbered by their associated RNA binding proteins (RBPs), can efficiently regulate the activity of interacting RBPs by driving their phase separation ${ }^{27}$. Discn was expressed at about $65-160$ copies per mouse ESC under genotoxic conditions (Supplementary Fig. 4j). Future works are required to clarify whether lncRNA-driven phase separation underlies Discn-mediated regulation.

Discn knockout mice show newborn death and brain dysfunctions. Stem cells are the cellular basis for embryo development and tissue homeostasis. Genomic instability in pluripotent stem cells or tissue stem cells could impair embryo development, cause premature ageing or cancer susceptibility. The phenotypes that we observed in cultured stem cells prompted us to further examine the physiological functions of Discn. To this end, we generated Discn knockout (KO) mice via CRISPR/Cas-mediated gene targeting strategy (Supplementary Fig. 6a). Two mouse lines from the same targeting design were established and the success of Discn KO was validated by Sanger sequencing of PCRamplified targeting regions (Supplementary Fig. 6b), as well as the loss of Discn expression in brain tissues of newborn $\mathrm{KO}$ mice (Supplementary Fig. 6c).

Heterozygous mutant mice $\left(\right.$ Discn $\left.^{+-}\right)$were viable and no obvious abnormality was observed. Crossings of $\mathrm{Discn}^{+/-}$mice in line 1 produced pups with expected Mendelian ratios (Fig. 6a). Similar results were obtained from mouse line 2 (Fig. 6a). To exclude a possible maternal effect of Discn on early embryonic development, we mated Discn ${ }^{-1}$ females with $\mathrm{Discn}^{+/-}$males. Pups were born in normal Mendelian ratios (Fig. 6b), confirming 
a

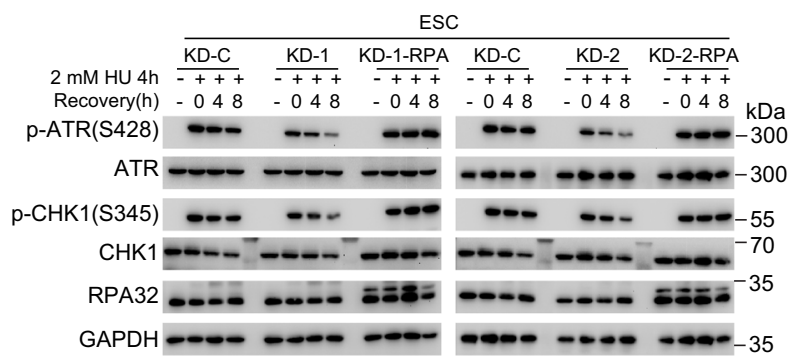

b

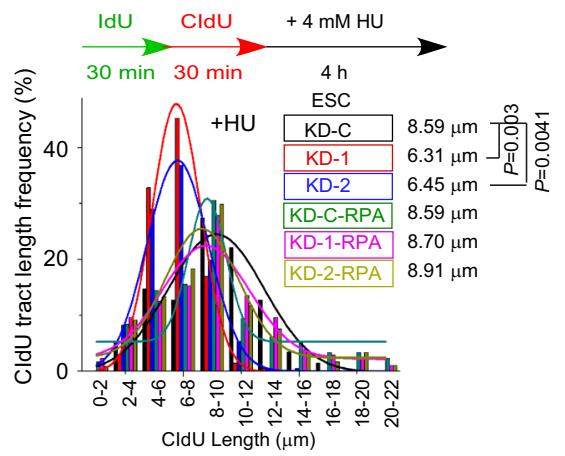

C

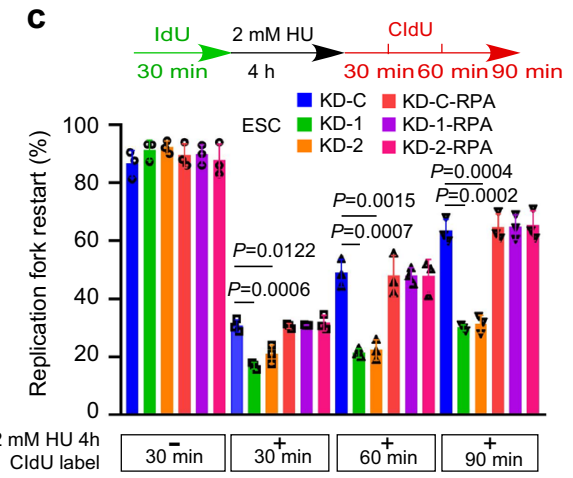

d $\frac{\mathrm{CldU}+/-0.1 \mathrm{mM} \mathrm{HU}}{10 \mathrm{~min}}$
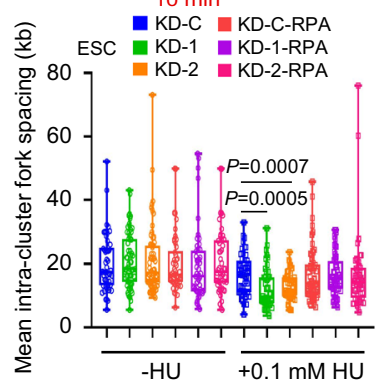

e

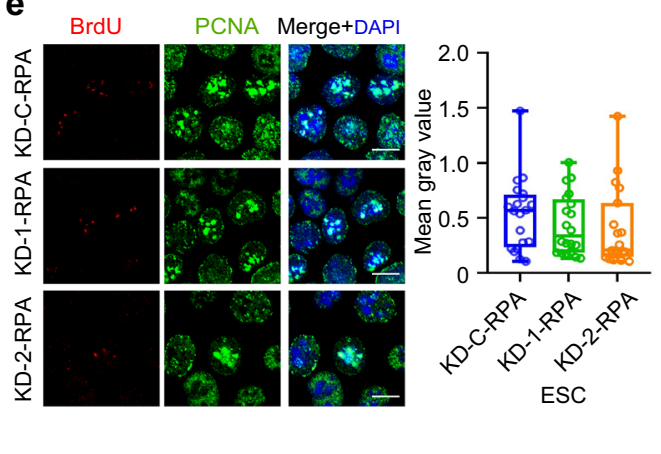

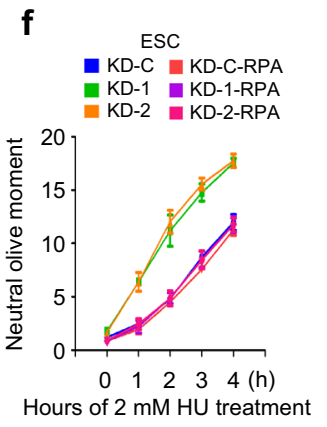
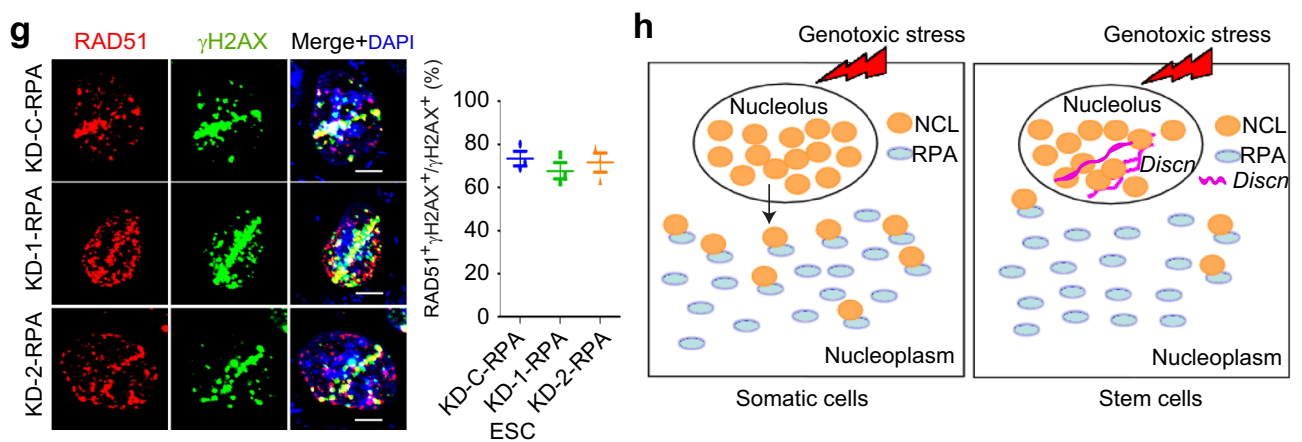

Fig. 5 RPA overexpression rescues genomic instability in Discn KD mESCs. RPA overexpression in Discn KD mESCs restored the ATR-CHK1 signaling (a), the stalled fork stability (b), and the fork restart ability (c). It also rescued the defects in dormant origin firing (d), ssDNA generation (e), and DSB formations (f) under HU treatment. In addition, RPA overexpression in Discn KD mESCs recovered the HR repair competence (g). Working model of Discn in safeguarding free RPA pool and genomic stability (h). Scale bar, $10 \mu \mathrm{m}$ in (e) and $5 \mu \mathrm{m}$ in (g). Three independent experiments were repeated with similar results in (a). At least 200 fibers from three independent replications were analyzed in (b, c). At least 50 continuous fibers from three independent replications were analyzed in (d). At least 19 images were analyzed in each condition in (e). Data were from three replications in (f, $\mathbf{g}$ ). Data were representative of individual values with box and whiskers plots showing the median, upper and lower quartiles, and minimum and maximum in (d, e). Data were presented as mean $\pm \mathrm{SEM}$ in $(\mathbf{c}, \mathbf{f}$ and $\mathbf{g}$ ). Statistical differences were determined using two-tailed Student's $t$-test.

that loss of Discn did not cause embryonic lethality. Consistently, crossing of Discn ${ }^{-1-}$ mice produced litters with grassly normal sizes (Fig. 6b). Thus, maternal-zygotic loss of Discn does not cause embryonic lethality. However, high rates of newborn death occurred in pups obtained from Discn ${ }^{-1-}$ crossings. More than half of them died within one week after birth (Fig. 6c).

We further investigated if Discn loss displayed long-term influences on the survived adults. Given that Discn was expressed in brain tissue and regulated the genomic integrity of cultured NSPCs, we thus focused on the brain functions. No structural defect was detected in brains of the Discn ${ }^{-1-}$ mice no matter whether they were from Discn ${ }^{-1-}$ or Discn ${ }^{-1-}$ crossings (Supplementary Fig. 6d). We then performed a series of behavioral tests to examine the emotions, learning, spatial memory, and sport performances. Adult littermates (two months old) from Discn ${ }^{+/}$crossings were utilized in these studies and significant differences were observed between wild-type (WT) and Discn ${ }^{-1-}$ mice. Discn ${ }^{-1-}$ mice displayed mood and anxiety-related disorders as determined by the following tests. In open field experiment, Discn ${ }^{-1-}$ mice spent significantly less time in the center (Fig. 6d) and crossed into the center for fewer times (Fig. 6e) than the WT littermates. Consistently, mutant mice stayed in the light box for shorter time in light-dark box test (Fig. 6f), and had more self-grooming behaviors (Fig. 6g, h) than WT littermates. We next conducted Morris water maze to evaluate the brain functions on learning and spatial memory. Discn ${ }^{-1-}$ mice and WT littermates swam in similar speeds (Supplementary Fig. 6e), and had equal ability to find the platform during the seven days of training (Supplementary Fig. 6f, g). However, in the following probe trials in which the escape platform was removed after training and the mice were allowed to search for it in a fixed time period, $\mathrm{Discn}^{-1-}$ mice displayed fewer platform crossings than WT littermates at $72 \mathrm{~h}$ rather than $4 \mathrm{~h}$ post training (Fig. 6i, Supplementary Fig. 6h). This result suggested the compromised long-term memory in Discn KO mice. We also performed rotarod test to examine the coordination 
a

\begin{tabular}{|c|c|c|c|}
\hline \multirow[b]{2}{*}{ Litter size } & \multicolumn{3}{|c|}{ Numbers of offspring } \\
\hline & $\mathrm{Discn}^{+/+}$ & $\mathrm{Discn}^{+/-}$ & Discn $^{-/-}$ \\
\hline Line $16.43 \pm 0.64(14)^{\star}$ & 18 & 49 & 23 \\
\hline Line $26.20 \pm 0.46(15)$ & 24 & 48 & 21 \\
\hline
\end{tabular}

* Litter size \pm SEM (number of litters)

C

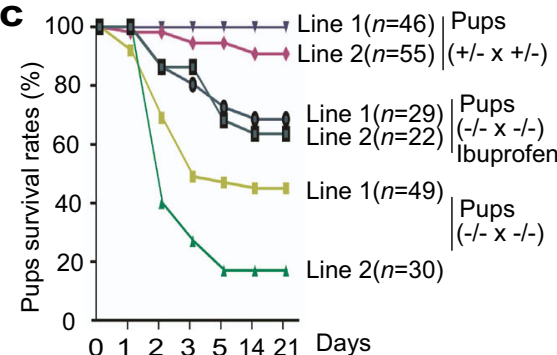

d

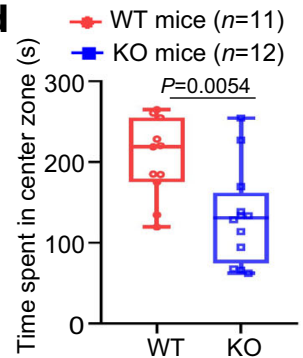

b

\begin{tabular}{|c|c|c|c|c|}
\hline \multicolumn{4}{|c|}{$\operatorname{Discn}^{+/-} \times$Discn $^{-/-}$(female) } & \multirow{3}{*}{$\frac{\text { Discn }^{-/-} \times \text {Discn }^{-/-}}{\text {Litter size }}$} \\
\hline \multirow[b]{2}{*}{ Lines } & \multirow[b]{2}{*}{ Litter size } & \multicolumn{2}{|c|}{ Vumbers of offspring } & \\
\hline & & Discn $^{-/-}$ & $\operatorname{Discn}^{+/-}$ & \\
\hline$\overline{\text { Line } 1}$ & $6.60 \pm 0.51(5)^{*}$ & 15 & 18 & $5.36 \pm 0.69(11)^{*}$ \\
\hline Line 2 & $7.00 \pm 0.58(4)$ & 18 & 10 & $6.67 \pm 0.80$ \\
\hline
\end{tabular}

* Litter size \pm SEM (number of litters) g

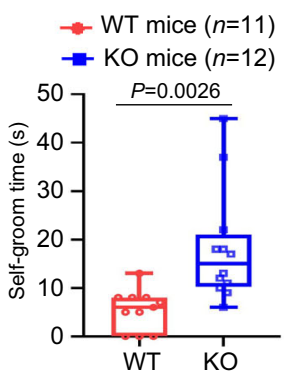

h $\rightarrow$ WT mice $(n=11)$

$\rightarrow$ KO mice $(n=12)$

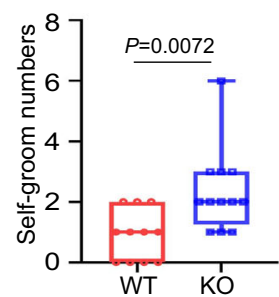

e $\quad-$ WT mice $(n=11)$

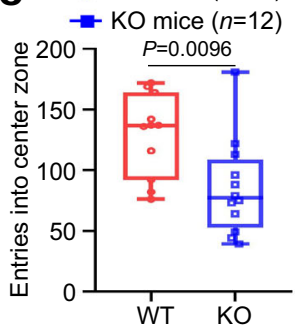

i

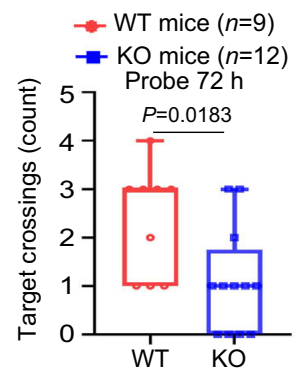

f $\quad \rightarrow$ WT mice $(n=11)$

$-\mathrm{KO}$ mice $(n=12)$
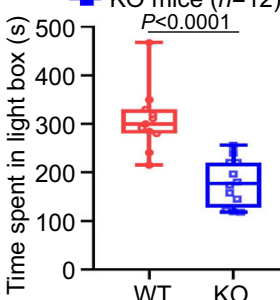

j

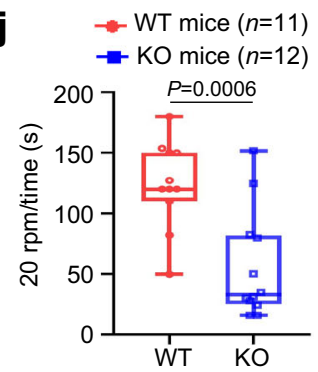

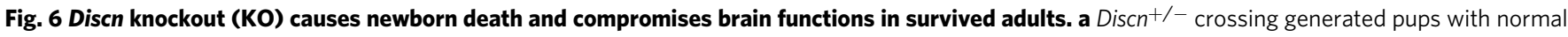
Mendelian ratios. b Discn- ${ }^{-1-}$ females mated with Discn ${ }^{+/-}$or Discn ${ }^{-/-}$males had normal litter sizes and Mendelian ratios. c Survival curve of offsprings from different mating strategies. Note that Discn ${ }^{-/-}$pups from Discn ${ }^{-/-}$crossings displayed newborn death, and administration of ibuprofen (0.4 mg/mL) to pregnant females alleviated the neonate death. Adult Discn $^{-/-}$mice and their WT littermates from Discn ${ }^{+/-}$crossings were utilized for behavioral tests. In open field test, Discn-/- mice spent less time in the center zone (d) and entered fewer times into the center zone (e) compared to the WT littermates. In light-dark box test, Discn-/- mice stayed in the light box for less time than WT littermates (f). In self-grooming behavior analysis, Discn-/- mice spent longer time ( $\mathbf{g}$ ) and showed more bouts (h) on self-grooming when compared to WT littermates. In Morris water maze test, at $72 \mathrm{~h}$ after 7 -day probe trials, Discn ${ }^{-/}$mice showed fewer platform crossings than WT littermates (i). In rotarod test, Discn-/- mice stayed on the rotating rod for shorter time than WT littermates (j). Data were representative of individual values with box and whiskers plots showing the median, upper and lower quartiles, and minimum and maximum in $(\mathbf{d}-\mathbf{j})$. Statistical differences were determined using two-tailed Student's $t$-test.

and balance abilities. Discn ${ }^{-1-}$ mice stayed on the rotating rod without falling for shorter time than WT littermates when the speed reached $20 \mathrm{rpm}$ (Fig. 6j), indicating the reduced coordination and balance. Because Discn $^{-1-}$ neonates generated from Discn $^{-1-}$ crossings displayed more severe phenotype than those generated from Discn ${ }^{+-}$crossings, we suspected that Discn $^{-1-}$ adults from $\mathrm{DisCn}^{-1-}$ crossings could have worse brain functions. To test this hypothesis, we compared their coordination and balance abilities. Indeed, Discn ${ }^{-1-}$ adults from Discn $^{-1-}$ crossings had worse performance than those from $\mathrm{Discn}^{+/-}$crossings in rotarod test (Supplementary Fig. 6i). Collectively, our data show that Discn plays important physiological functions and its loss in mice causes newborn death as well as brain dysfunctions in survived adults.

Discn $^{-/-}$mouse brains have massive DNA DSBs and inflammation. NSPCs are the cellular basis for neurogenesis at fetal and postnatal stages. Accumulations of DNA DSBs and cytosolic double-strand DNA (dsDNA) in NSPCs and their progenies not only impaired neurogenesis and brain functions ${ }^{28,29}$, but also evoked innate immune and inflammation responses leading to animal death ${ }^{30}$. Based on the function of Discn in in-vitro cultured NSPCs, we hypothesized that Discn KO might cause accumulations of DSBs and cytosolic dsDNA in brain cells of neonates and adults, and the damages and associated immune responses underlie the newborn death and brain dysfunctions. Indeed, the brain cells of Discn ${ }^{-1-}$ pups (one day old) contained higher level of DNA DSBs measured by $\gamma \mathrm{H} 2 \mathrm{AX}$ staining and neutral comet assay (Fig. 7a, b), and cytosolic dsDNA (Fig. 7c) compared to WT pups. Notably, KO pups from Discn ${ }^{-1-}$ crossings had more abundant DSBs and cytosolic dsDNA than those from Discn ${ }^{+1-}$ crossings (Fig. 7b, c). Similar results were obtained from adult mouse brains (Supplementary Fig. 7a-c).

DNA DSBs and cytosolic dsDNA can activate the STING pathway inducing the type 1 interferon expression and downstream immune responses ${ }^{31-33}$. Cytosolic dsDNA also stimulates the AIM2 inflammasome activation leading to caspase 1 (CASP1) cleavage, pro-interleukin-1 $\beta$ (IL-1 $\beta$ ) maturation and cell death via gasdermin D-mediated pyroptosis ${ }^{31,34}$. STING pathway activation is monitored by phosphorylation of the key components TBK1 and IRF3, and the expression of type 1 interferons (INFa and INF $\beta$ ). Compared to the WT counterparts, adult Discn KO mice, in particular those from Discn ${ }^{-1-}$ crossings, showed drastic STING activation in brain cells (Fig. 7d). Moreover, cleavage of CASP1 and maturation of IL- $1 \beta$ were elevated in $\mathrm{KO}$ mouse brains (Fig. 7e). Intriguingly, the DNA DSBs and immune reactions were not detected in lung tissue of $\mathrm{KO}$ mice (Supplementary Fig. 7d). This suggested that the influences of Discn loss might be confined to certain tissues/organs where tissue stem cells rely on Discn for genome stability. Severe immune and inflammation reactions usually cause animal death. We then asked if the genomic instability-driven inflammation accounted for the newborn death of Discn KO mice. To this end, 
a

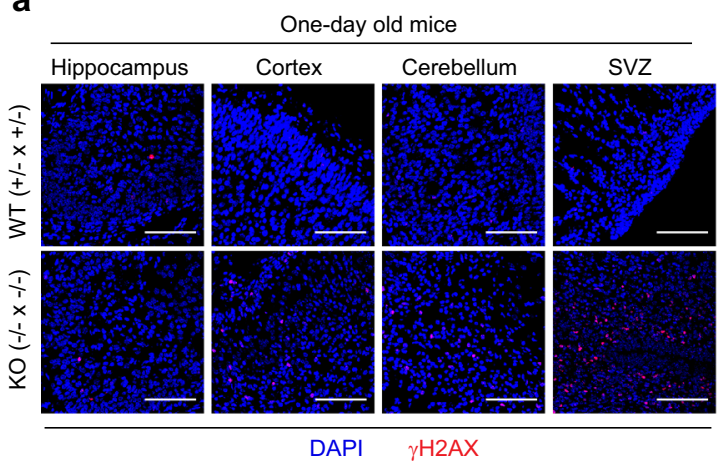

b

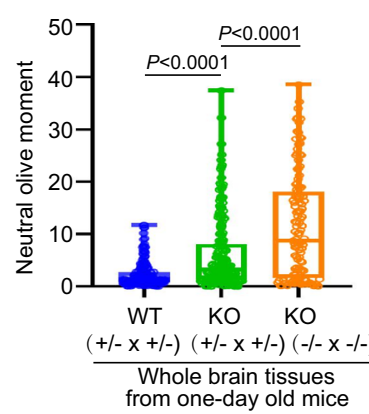

C

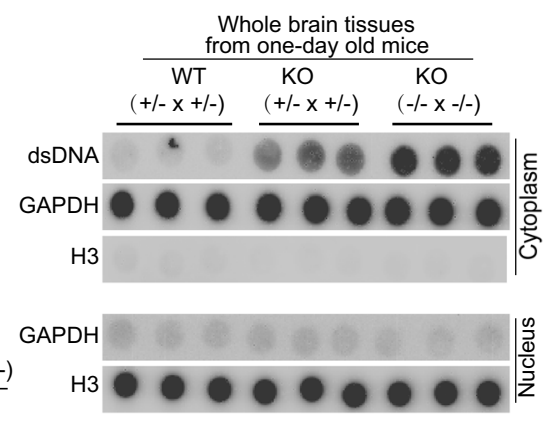

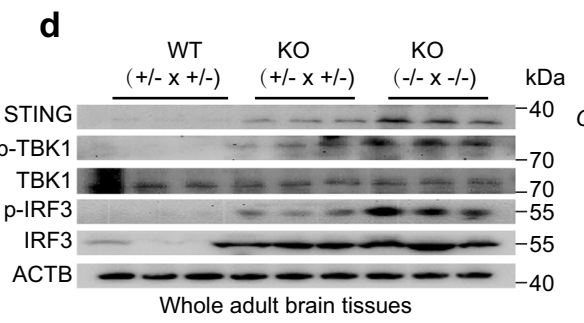

f

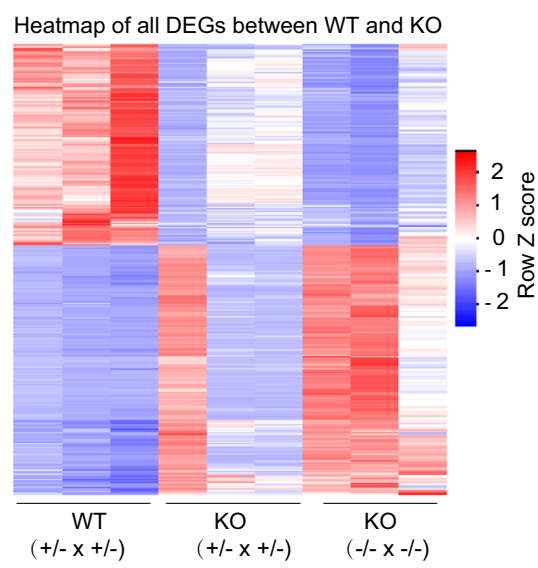

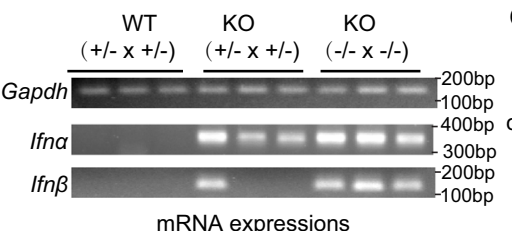

mRNA expressions

Whole adult brain tissues

g

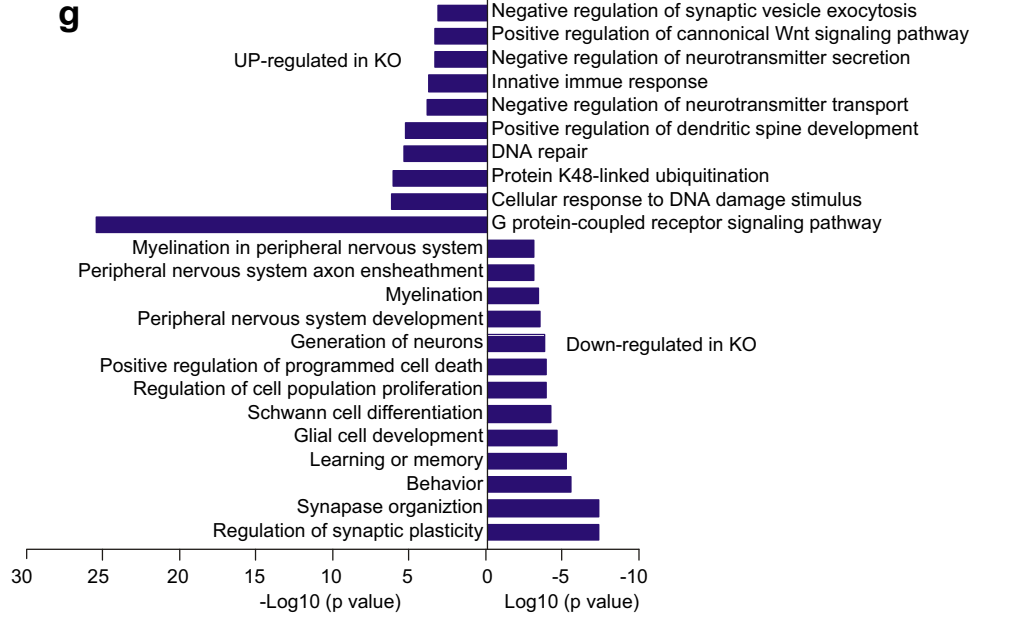

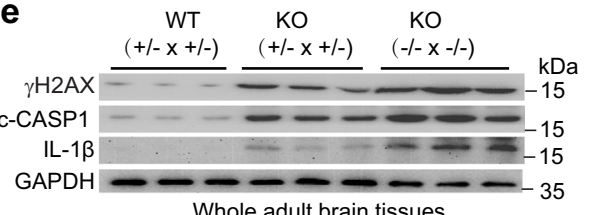

Whole adult brain tissues

Positive regulation of autophagy

Positive regulation of autophagy

Tegative regulation of synaptic vesicle exocytosis

Positive regulation of cannonical Wnt signaling pathway

Negative regulation of neurotransmitter secretion

ve immue respons

Generation of neuron

Fig. 7 Discn knockout (KO) in mice induces massive DNA DSBs and immune reaction. Discn KO mouse brains contained higher level of DNA DSBs than WT counterparts, as indicated by $\gamma \mathrm{H} 2 \mathrm{AX}$ staining (a) and comet assay (b). SVZ, subventricular zone. $\mathrm{KO}(+/-\times+/-)$ indicated the $\mathrm{KO}$ mice were from Discn $^{+/-}$crossing, whereas KO $(-/-\times-/-)$indicated the KO mice were from Discn ${ }^{-/-}$crossing. Scale bar, $100 \mu \mathrm{m}$. At least 100 tails were analyzed in each comet assay group in three independent replications. Data were representative of individual values with box and whiskers plots showing the median, upper and lower quartiles, and minimum and maximum. Two-tailed Student's t-test. c Discn KO mouse brains contained higher level of cytosolic dsDNA than WT mice. Mice were 1 day old in (a-c). d The STING pathway was activated in Discn KO mouse brains, as indicated by the phosphorylation of TBK1 and IRF3, as well as the induction of type 1 interferon expression. Three independent experiments were repeated with similar results. $\mathbf{e}$ The inflammasomes were activated in Discn KO mouse brains, as indicated by the detection of cleaved caspase 1 (c-CASP1) and mature IL-1 1 . Three independent experiments were repeated with similar results. $\mathbf{f}$ Heatmap of differential expressed genes (DEGs) between Discn KO and WT counterpart mice brains. $\mathbf{g}$ Gene ontology (GO) enrichments of DEGs shown in (f). Adult mice were used in $(\mathbf{d}-\mathbf{g})$.

we administrated ibuprofen, which is able to alleviate inflammation, in drinking water to pregnant $\mathrm{Discn}^{-1-}$ females mated with $\mathrm{Discn}^{-1-}$ males ${ }^{35}$. Notably, ibuprofen administration partially rescued the defect of newborn death (Fig. 6c), supporting that inflammation is a causative factor for animal death.

We finally examined the whole-genome gene expression changes in Discn KO mouse brains by RNA sequencing. Brain tissues of WT and $\mathrm{DisCn}^{-1-}$ mice, which were derived from crossings of Discn ${ }^{+/-}$ and Discn $^{-1-}$, respectively, were analyzed. We identified 3535 differentially expressed genes (DEGs) whose expressions were consistently decreased or increased (fold change $\geq 2$ ) in two types of Discn KO mice compared to the WT control (Fig. 7f, Supplementary data). Of note, Discn ${ }^{-1-}$ mice born from Discn ${ }^{-1-}$ crossings displayed the most drastic changes when compared to the other groups. Gene Ontology (GO) term analyses revealed that DEGs down-regulated in Discn $^{-1-}$ were mostly involved in the regulations of neurogenesis, neuronal activities and functions (Fig. $7 \mathrm{~g}$, Supplementary Fig. 7e, f). DEGs upregulated in Discn ${ }^{-1-}$ mice were enriched in terms of negative regulations of neurogenesis and neuronal functions, DNA damage responses, positive regulation of autophagy and cell differentiation (Fig. 7g, Supplementary Fig. 7g). Consistently, term of innate immune response was enriched (Fig. 7g, Supplementary Fig. 7h, i).

\section{Discussion}

Stem cells are the cellular basis for embryo development and tissue homeostasis, and are known to have much more stable 
genome than differentiated somatic cells. Only recently was it recognized that stem cells employed unique DNA metabolic pathways to efficiently maintain stable genome. Although several pioneer works reported stem-cell specific regulations on telomere lengthening, DNA damage response, and repair ${ }^{2-7,36}$, it remains largely unknown regarding the specificity of DNA metabolism and their relevance to genomic stability in stem cells. LncRNAs play increasingly important functions in many cellular events. Whether lncRNAs are involved in stem-cell specific regulations of DNA metabolism is unexplored. In this study, we identified a list of ESC-specific lncRNAs whose expressions are responsive to genotoxic stress. By analyzing one un-annotated lncRNA Discn, we identified a novel regulatory pathway Discn-NCL-RPA which preserves a sufficient RPA pool for DNA replication stress response and repair.

During continuous cell proliferation, DNA replication frequently encounters endogenous and exogenous barriers which stall the replication forks and generate replication stress. During stress, ssDNA is persistently generated due to the continuous firing of new origins even the checkpoint is active. The ssDNA is not converted into DNA breaks as long as it is coated and protected by RPA. Thus, the RPA surplus plays a central part and is considered as a common denominator for avoiding replication catastrophe $e^{25,26}$. Cells usually express more RPA than needed during the normal unperturbed replication. However, excessive ssDNA generated by replication stress can rapidly exhaust RPA reservoir $^{26}$. Therefore, efficient sustaining the RPA pool is vital to survive the replication stress. Increasing the expression of RPA is one simple strategy that is employed by pluripotent stem cells. Here we reported a novel mechanism in which lncRNA Discn sustains the free RPA pool by sequestering NCL in nucleolus and counteracting its association with RPA. The Discn-NCL binding in nucleolus seems cell autonomous and does not require stress stimuli. Discn is induced by genotoxic stress and its expression level is adjusted by the strength of stress (for instance hydroxyurea treatment plus ATR deficiency). Therefore, Discn finetunes the RPA reservoir in response to replication stress.

Discn depletion causes severe DNA DSBs in cultured mouse ESCs which are in vitro counterparts of the undifferentiated epiblast cells in peri-implantation embryos. Previous study reported that induction of DSBs at gastrulation stage (E6.5-E7.5) could result in apoptosis and embryonic lethality ${ }^{37}$. However, Discn KO did not cause prenatal death immediately after gastrulation. This could be due to the fact that the pluripotent epiblast cells exist transiently in vivo (from embryonic E4.5-E5.5) and undergo limited rounds of DNA replication during this timewindow. The extent of DNA replication-associated DSBs could therefore be mild and fail to evoke lethal phentotype. In contrast, NSPC-based neurogenesis starts from $\sim$ E8.5 and persists throughout the fetal stage and even until after birth. The long time peroids of NSPC proliferation renders the neural system susceptible to the replication-associated DNA damage. Indeed, we detected increased levels of DNA DSB and cytoplasmic dsDNA in brain cells of Discn KO mice. Moreover, the DSB level was significantly higher in $\mathrm{KO}$ mice derived from $\mathrm{Discn}^{-1-}$ crossings than in those derived from Discn ${ }^{+/-}$crossings. Concordantly, Discn KO mice displayed variable extents of developmental defects ranging from brain dysfunctions to newborn death, depending on the genotypes of their parents.

The genomic instability acted as a causative factor in the developmental defects of Discn KO mice. Previous studies including ours have shown that in NSPCs, replication stressinduced DNA DSBs frequently arise in replication fragile sites and form recurrent DSB clusters (RDCs). Many genes critical for neurogenesis in NSPCs harbor RDCs and are susceptible to replication stress-associated perturbations. Thus, replication- associated DNA DSBs in NSPCs directly impacted neurogenesis $29,38,39$. In addition, DNA DSBs and in particular cytoplasmic dsDNA are able to evoke innate immune response and inflammatory reactions ${ }^{33,40}$. Immune reaction alone in developing brain imparts lifelong neuropathology and altered behaviors ${ }^{41}$. Adult neural stem cells are also altered by systemic inflammation ${ }^{42}$. Severe inflammation is able to cause pyroptotic cell death ${ }^{31,34}$. Indeed, Discn KO induced the STING pathway activation leading to the production of type-1 interferons. Inflammasomes were also activated in KO brains as evidenced by the cleavage of caspase 1 and maturation of pro-inflammatory cytokine IL-1 $\beta$. Similar to the tendency of DNA DSB and cytoplasmic dsDNA accumulation, the immune and inflammatory reactions in $\mathrm{KO}$ mice from Discn $^{-/-}$crossings were more severe, leading to the newborn death. It is intriguing to know why $\mathrm{KO}$ mice from $\mathrm{Discn}^{-1-}$ crossings accumulated more DNA DSBs than those from Discn ${ }^{+/}$crossings. One possible explanation is that oocytes and sperms from Discn ${ }^{-1-}$ mice might contain more DSBs, which heightens genomic instability of embryos ${ }^{43}$.

In summary, our study uncovered a novel stem cell-specific pathway Discn-NCL-RPA which regulates RPA availability and genomic stability, and plays physiological role in mouse neurogenesis. Our attempts to identify the tissue expression pattern of human DISCN using publicly available RNA-seq data were unsuccessful. Future works are needed to investigate the human DISCN expression patterns and functions, and its relevance to human diseases.

\section{Methods}

Mouse embryonic fibroblasts (MEF) preparation and transfection. MEF was isolated from embryos of CD1 mice at embryonic day 13.5 (E13.5) as previously described ${ }^{44}$. Briefly, pregnant mice were euthanized and the embryos were dissected. The fetus head and internal organs were removed. Then the fetus tissue was minced thoroughly and digested with $3 \mathrm{~mL}$ of $0.25 \%$ trypsin-EDTA at $37^{\circ} \mathrm{C}$ for 10 min. Tripsin was neutralized with DMEM (Gibco, 11965) supplemented with $10 \%$ FBS (Gibco, $10099141 \mathrm{C}$ ). The suspension was transferred to a $50 \mathrm{~mL}$ conical tube to allow the undissociated tissues pellet with gravity for $10 \mathrm{~min}$. MEF in supernatant was transferred to $100 \mathrm{~mm}$ dishes for further culture. MEF was cultured in DMEM supplemented with 10\% fetal bovine serum. To prepare feeder cells for ESC culture, MEF was expanded for three passages and treated with $5 \mu \mathrm{g} /$ $\mathrm{mL}$ mitomycin C (Sigma, M4287) for $4 \mathrm{~h}$. Inactive MEF was frozen in liquid nitrogen. For ectopic expression of Discn in MEF, cells were transfected with lipofectamine 2000 according to the manufacturer's instructions.

Mouse ESC culture and induced differentiation. Mouse ESCs (derived in our lab) were routinely maintained on inactivated MEF in the following culture medium: Dulbecco's Modified Eagle Medium/Nutrient Mixture F-12 (DMEM/F12) medium supplemented with $20 \%$ Knockout serum replacement (Gibco, 10828028), $1 \mathrm{mM}$ sodium pyruvate (Gibco, 11360070), 2 mM L-glutamine (Sigma, G8540), 1\% non-essential amino acids (Gibco, 11140-035), $0.1 \mathrm{mM} \beta$-mercaptoethanol (Sigma; M7522), penicillin (100 U/mL)/streptomycin (100 $\mu \mathrm{g} / \mathrm{mL})(\mathrm{Gibco}, 15140-122)$ and 1000 units/mL mouse leukemia inhibitory factor (LIF) (Millipore, ESG1107). For ATR inhibition, cells were treated for $4 \mathrm{~h}$ with $10 \mu \mathrm{M}$ VE-821 (Selleck, S8007).

To obtain differentiated cells, mouse ESCs were passaged with $0.05 \%$ TripsinEDTA (Gibco, 25200072) and seeded onto $0.1 \%$ gelatin-coated dishes twice to remove feeders. Culture medium was replaced with differentiation medium: culture medium without LIF and supplemented with $0.5 \mu \mathrm{M}$ retinoic acid (Sigma, R2625). After one week induction, the differentiated cells were harvested for RNA extraction.

Neural stem/progenitor cell (NSPC) culture. Mouse NSPC was previously derived from WT mice at postnatal day 5 (P5) ${ }^{29}$. Cells were seeded in $35-\mathrm{mm}$ dishes pre-coated with Laminin (Sigma, L2020) and maintained in complete medium (STEMCELL Technologies, 05702) supplemented with $10 \mathrm{ng} / \mathrm{mL}$ basic fibroblast growth factor (bFGF) (STEMCELL Technologies, 02634), $20 \mathrm{ng} / \mathrm{mL}$ recombinant human epidermal growth factor (rhEGF) (STEMCELL Technologies, 02633), and $2 \mu \mathrm{g} / \mathrm{mL}$ heparin (STEMCELL Technologies, 07980).

Generation of Discn Knockout mice. The Discn ${ }^{+/-}$mouse strain was generated on the C57Bl/6J genetic background by Cyagen Company (https://www.cyagen.com). Briefly, a pair of gRNA located on both sides of mouse Discn gene and Cas9 mRNA were co-injected into mouse zygotes to generate targeted knockout offsprings. The gRNA target sequences were CAATTCATCGGATTTCTGAGAGG and ATAGAGG 
CACAGTAGATTCGGGG. Discn knockout was validated by Sanger sequencing of PCR-amplified fragment. Genotying primers, P1, P2-Mut, and P3-WT, were listed in Supplementary Table 1. PCR-based genotyping was performed using genomic DNAs from mouse tails at the following program: $94^{\circ} \mathrm{C}$ for $5 \mathrm{~min}$, followed by 35 cycles of denaturation at $94^{\circ} \mathrm{C}$ for $15 \mathrm{~s}$, annealing at $64^{\circ} \mathrm{C}$ for $30 \mathrm{~s}$, and extension at $68^{\circ} \mathrm{C}$ for $36 \mathrm{~s}$.

Mice were housed in accordance to the Animal Care and Use Committee rules and guidelines of the Kunming Institute of Zoology, Chinese Academy of Sciences. Mice were kept under $12 \mathrm{~h} \mathrm{dark/light} \mathrm{cycle,} \mathrm{with} \mathrm{daylight} \mathrm{from} \mathrm{8:00} \mathrm{to} \mathrm{20:00,}$ ambient temperature 20 to $25^{\circ} \mathrm{C}$, and 40 to $70 \%$ humidity. All works on mice were carried out in accordance with the guidelines of the Animal Care and Use Committee of the Kunming Institute of Zoology, Chinese Academy of Sciences. All the studies on mice received ethical approval of the Animal Care and Use Committee of the Kunming Institute of Zoology, Chinese Academy of Sciences.

RNA sequencing and data analyses. Total RNAs were extracted from cultured cells or from mouse brains using Trizol (Tiangen, DP424). The cDNA libraries were constructed using the TruSeq ${ }^{\mathrm{TM}}$ RNA Sample Preparation Kit (Illumina) and sequenced using an Illumina HiSeq 3000 or HiSeq X Ten platform. To identify novel lncRNAs, the clean reads were mapped to mouse reference genome (mm9) using Tophat2 software (Version 2.0.8). Transcripts were reassembled by Cufflinks (Version 2.1.1 $)^{45}$. Non-coding transcript annotation file was downloaded from the NONCODE database (http://www.noncode.org/) (Version 4.0). Novel lncRNAs were identified by Coding-Non-Coding Index (CNCI) software (Version 2) ${ }^{46}$. Differentially expressed lncRNAs were determined by DEGseq software (Version 1.38.0 $)^{47}$.

For coding gene analysis, the clean reads were mapped to mouse reference genome (mm10) using Tophat2 software (Version 2.0.8). The values of gene expression were calculated by Cufflinks (Version 2.1.1) ${ }^{45}$. Differentially expressed genes were determined using Cuffdiff software (Version 2.1.1). Gene ontology enrichment was performed using an online tool (http://geneontology.org/) 48,49 The heatmaps were created by the "gplots" R packages with default parameter. The RNA-seq data have been deposited in the Gene Expression Omnibus (GEO) database (accession number: GSE161998).

Rapid amplification of CDNA ends (RACE). The RACE protocol is based on published Smart-seq2 protocol ${ }^{50}$. The oligo-dT primer, template-switching oligos (TSO) and ISPCR primers were synthesized as described in the Smart-seq2 method. For $5^{\prime}$ end amplification of Discn, reverse transcription was performed using gene specific primer 1 (GSP1). TSO primer was used for template switching. $5^{\prime}$ end was amplified by PCR using gene specific primer 1 and ISPCR primer. For $3^{\prime}$ end of Discn, reverse transcription was performed using oligo-dT primer. $3^{\prime}$ end was amplified by PCR using gene specific primer 2 (GSP2) and ISPCR primer. The products were cloned into pEasy-blunt vector (Transgene) and validated by the Sanger sequencing. The primers used for PCR were listed in Supplementary Table 1.

Expression vector construction. To construct the shRNA expression plasmids, shRNA oligos targeting to Discn were designed through an online tool of Ambion siRNA Target Finder. The nucleotide sequences, shRNA-control, shRNA\#1, shRNA\#2, were listed in Supplementary Table 1. The shRNA oligos were synthesized by Tsingke Company (Beijing, China), annealed and inserted into pLKO.1 plasmid using EcoR I and Age I sites.

For Discn-expression plasmids construction, full length Discn or predicted open reading frames (ORFs) tagged by FLAG at $3^{\prime}$ end were separately amplified using standard PCR procedure and inserted into pCDNA3.1(+) plasmid with the following primers: pCDNA3.1-Discn, ORF-1 Discn, ORF-2 Discn, ORF-3 Discn, ORF-4 Discn, ORF-5 Discn, ORF-6 Discn, and ORF-7 Discn. For construction of Discn or Discn mutants inducible expression plasmids, Discn or Discn mutants were inserted into PTRIPZ vector which carries Tet ON promoter using the following primers: Inducible-Discn, Discn $5^{\prime}$ end, Discn $3^{\prime}$ end, and Discn central part. Expression vectors were packaged into lentivirus for transfection.

pTomo-RPA70-P2A-RPA32-P2A-RPA14 fusion construct was made as followings. Coding sequences for three RPA subunits (RPA70, RPA32, and RPA14) were obtained from cDNA transcribed from mRNA of mESCs by standard PCR procedures with the primers RPA70-p, RPA32-p, and RPA14-p. The RPA fragments were joined with $\mathrm{P} 2 \mathrm{~A}$ using overlap PCR with the following primers: RPA joint primer, RPA70-P2A primer, P2A-RPA32-P2A primer, and P2A-RPA14primer. The fragment containing RPA70-P2A-RPA32-P2A-RPA14 was then ligated into pTomo plasmid. The primers used for PCR were listed in Supplementary Table 1.

Lentivirus package and cell transfection. HEK293T cells were seeded in $15-\mathrm{cm}$ dishes for $24 \mathrm{~h}$, co-transfected with $12 \mu \mathrm{g}$ expression vector (pLKO.1 or pTRIPZ or pTomo), $6 \mu \mathrm{g}$ pSPAX, and $6 \mu \mathrm{g}$ pM2D 2.0 plasmid using polyethyleneimine (PEI) (Sigma). After transfection for $16 \mathrm{~h}$, the medium was replaced by fresh DMEM with $10 \%$ FBS. The viral supernatants were collected at $48 \mathrm{~h}$ and $72 \mathrm{~h}$ post-transfection, respectively. The viruses were filtered through a $0.45 \mu \mathrm{M}$ syringe filter and concentrated through an Amicon ${ }^{\circledast}$ Ultra-15 Centrifugal Filter (Millipore, R9EA11841)

For Discn knockdown or inducible expression, cells were transducted with virus and $8 \mu \mathrm{g} / \mathrm{mL}$ polybrene, and selected with $0.5 \mu \mathrm{g} / \mathrm{mL}$ puromycin.

Metaphase spread preparation. Cells were treated with $0.02 \mu \mathrm{g} / \mathrm{mL}$ colcemid (Sigma, C3915) for $2 \mathrm{~h}$ to arrest in metaphase. Cells were then harvested, resuspended in hypotonic buffer $(0.4 \%$ sodium citrate and $0.4 \% \mathrm{KCL})$ and incubated for $15 \mathrm{~min}$ at $37^{\circ} \mathrm{C}$. After cell swelling, $2 \mathrm{~mL}$ freshly prepared methanol-acetic acid fixative (3:1) was added into the hypotonic buffer. Cells were pelleted by centrifuging for $5 \mathrm{~min}$ at $200 \mathrm{~g}$. The pellet was re-suspended in $5 \mathrm{~mL}$ of 3:1 methanolacetic acid for $20 \mathrm{~min}$ and dropped onto slides to obtain metaphase spreads. At least 50 metaphases were analyzed in three independent replications.

Sister chromatid exchange (SCE) assay. SCE assay was performed as previously described ${ }^{6,51}$. Briefly, $1 \times 10^{6}$ mouse ESCs were cultured without feeder in ESC medium supplemented with $20 \mu \mathrm{M}$ bromodeoxyuridine (BrdU, Sigma) for $24 \mathrm{~h}$. In the final $2 \mathrm{~h}$, cells were treated with $0.02 \mu \mathrm{g} / \mathrm{mL}$ colcemid. For SCE assay of mouse NSPCs, cells were cultured in NSPC medium adding $20 \mu \mathrm{M}$ BrdU for $48 \mathrm{~h}$. In the final $4 \mathrm{~h}$, NSPCs were incubated with $0.02 \mu \mathrm{g} / \mathrm{mL}$ colcemid. Cells were harvested and re-suspended in hypotonic buffer $(0.4 \%$ sodium citrate and $0.4 \% \mathrm{KCL})$ for $15 \mathrm{~min}$ at $37^{\circ} \mathrm{C}$. The swollen cells were fixed in freshly prepared methanol-acetic acid fixative (3:1) for $30 \mathrm{~min}$ and dropped onto ice-cold wet slides. The slides were dried at $65{ }^{\circ} \mathrm{C}$ overnight. The dried slides with metaphase spreads were immersed in $10 \mu \mathrm{g} / \mathrm{mL}$ of Hoeschst 33258 for $20 \mathrm{~min}$ and washed with $2 \times$ SSC buffer $(0.3 \mathrm{M}$ $\mathrm{NaCl}, 0.03 \mathrm{M}$ sodium citrate; $\mathrm{pH} 7.0$ ) once. Then the slides covered with lens paper were exposed coverslip-side-up in $55^{\circ} \mathrm{C} 2 \times$ SSC buffer to $365 \mathrm{~nm}$ UV light at a distance of $1 \mathrm{~cm}$ for $30 \mathrm{~min}$. The slides were immersed in $1 \times$ SSC and incubated for $1 \mathrm{~h}$ at $50^{\circ} \mathrm{C}$. The slides were stained with $10 \%$ Giemsa solution (Gibco, 1892798) for $10 \mathrm{~min}$ at $37^{\circ} \mathrm{C}$. After washing with water and dried overnight at room temperature, SCEs images were captured using an Olympus confocal microscopy system.

Neutral comet assay. The neutral comet assay was performed as previously described ${ }^{6}$. Briefly, cells were dissociated into single cells at the concentration of $1 \times 10^{5}$ cells $/ \mathrm{mL}$. Cells were then mixed with low-melting agarose at a ratio of $1: 8$ and spread onto the slides. Cells were lyzed in lysis buffer $(2.5 \mathrm{M} \mathrm{NaCl}, 100 \mathrm{mM}$ EDTA, $10 \mathrm{mM}$ Tris, $1 \% \mathrm{~N}$-lauroylsarcosine, and $1 \%$ TritonX-100) at room temperature for $1 \mathrm{~h}$. Electrophoresis was performed in electrophoresis buffer $(300 \mathrm{mM}$ sodium acetate, $100 \mathrm{mM}$ Tris, $\mathrm{pH}=8.3$ ) for $30 \mathrm{~min}$ at $80 \mathrm{~mA}$. DNA was fixed in $100 \%$ ethanol and stained with DAPI $(10 \mathrm{ng} / \mathrm{mL})($ Thermo). The comet tails were analyzed by Komet 7 comet assay software (Andor Technology). At least 100 cells were analyzed per group. Three independent replications were performed.

Immunofluorescence staining in cells. For immunofluorescence staining, cells were fixed in $4 \%$ paraformaldehyde (PFA), permeabilized in $0.5 \%$ Triton X-100, blocked in $2 \%$ BSA for $2 \mathrm{~h}$ and incubated with primary antibodies at $4{ }^{\circ} \mathrm{C}$ overnight. After washing with $0.2 \%$ Tweeen 20/PBS three times at room temperature, cells were incubated with secondary antibodies at room temperature for $1 \mathrm{~h}$. Cells were washed three times with PBS and counterstained with DAPI for $10 \mathrm{~min}$. After washing with PBS three times again, slides were sealed with snail oil. Antibody information was listed in Supplementary Table 2.

Brain section, hematoxylin and eosin (H\&E) staining, and immunostaining After induction of deep anesthesia, mice were transcardially perfused with ice-cold phosphate-buffered saline (PBS) followed with $4 \%$ paraformaldehyde (PFA) in PBS. Then the brains were harvested and post-fixed in $4 \%$ PFA overnight at $4{ }^{\circ} \mathrm{C}$ After sunk in 15 and 30\% sucrose in PBS, the brains were embedded in OCT (optimal cutting temperature compound) for sectioning. H\&E staining and immunostaining were performed following the standard protocol.

Nucleolus and nucleoplasm fractionation. Nucleoli were isolated as previously described ${ }^{52}$. Briefly, $2 \times 10^{7}$ cells were harvested and resuspend in ice-cold hypotonic buffer $(10 \mathrm{mM} \mathrm{NaCl}, 1 \mathrm{mM} \mathrm{MgCl}, 10 \mathrm{mM}$ Tris.HCl, $\mathrm{pH}=7.4)$ supplemented with Roche complete protease inhibitor (Roche Applied System) and Ribonucleoside Vanadyl Complex (10 mM) (New England BioLabs, S1402S) to have cytoplasm detach from nuclei. Cells were lysed by a $0.45-\mathrm{mm}$ clearance $1-\mathrm{mL}$ Dounce homogenizer. Nuclei were purified and broken by sonication in ice-cold water. Nucleoli were then purified through a sucrose cushion.

Nucleoplasm fractionation was performed as previously described ${ }^{53}$. Briefly, cells were washed once with ice-cold PBS, and then fixed in $1 \%$ formaldehyde for $15 \mathrm{~min}$. Cells were lyzed in lysis buffer $(25 \mathrm{mM}$ Tris, $\mathrm{pH}=7.4,0.1 \%$ Triton X-100, $85 \mathrm{mM} \mathrm{KCl}$ ). The nuclei were pelleted by centrifuging for $5 \mathrm{~min}$ at $2300 \times \mathrm{g}$. Then the nuclei were lyzed in SDS buffer $(50 \mathrm{mM}$ Tris $\mathrm{pH}=7.4,10 \mathrm{mM}$ EDTA, $4 \%$ SDS). After centrifuging at $16,000 \times g$ for $30 \mathrm{~min}$, the supernatant was collected as nucleoplasm. 
RNA fluorescence in situ hybridization (FISH) and immunostaining. For RNAFISH of Discn, DNA probes and RNA-FISH hybridization kit were obtained from Guangzhou RiboBio Co., LTD. FISH was performed according to the manufacturer's protocol with small modification. Briefly, cells were grown on glass covers pre-coated by Matrigel (BD), permeabilized with CSKT buffer [10 $\mathrm{mM}$ PIPES ( $\mathrm{pH}=6.8$ ), $100 \mathrm{mM} \mathrm{NaCl}, 3 \mathrm{mM} \mathrm{MgCl} 2,0.3 \mathrm{M}$ sucrose and $0.5 \%$ Triton X100 ] at $4{ }^{\circ} \mathrm{C}$ for $10 \mathrm{~min}$ and fixed in $4 \%$ paraformaldehyde at room temperature for $10 \mathrm{~min}$. Hybridization was performed at $37^{\circ} \mathrm{C}$ overnight. Glass covers were washed for 5 min three times with $4 \times$ saline sodium citrate buffer (SSC), once with $2 \times$ SSC, once with $1 \times$ SSC at $42^{\circ} \mathrm{C}$ and once with PBS at room temperature. Immunostaining was performed as described in section of immunofluorescence staining for cells.

Cell proliferation assay. To examine the proliferation, dividing cells were labeled with $10 \mathrm{mM} \mathrm{BrdU}$ for $30 \mathrm{~min}$ followed by fixation in $4 \%$ formaldehyde for $10 \mathrm{~min}$ and denaturation in $2 \mathrm{M} \mathrm{HCl}$ at room temperature for $1 \mathrm{~h}$. After staining with antiBrdU primary antibody (Novus) and $\mathrm{Cy} 3$ conjugated secondary antibody, the $\mathrm{BrdU}^{+}$cells were sorted by using a FACS LSRFortessa flow cytometer (BD) and BD FACSDiva software (Version 8.0.2). Data were analyzed with Flowjo software (Version 7.6). The gating strategy for $\mathrm{BrdU}^{+}$cells was presented in Supplementary Fig. 8.

In vitro RNA pulldown and mass spectrometry analysis. RNA was in vitro transcribed with T7 RNA polymerase (Fermentas, EP0111), and was labeled with biotin using Pierce ${ }^{\mathrm{TM}}$ RNA 3' End Desthiobiotinylation Kit (Thermo, 20163) according to the manufacturer's instructions. In vitro RNA pulldown was performed as previously described ${ }^{54}$. Breifly, 50 pmol of labeled RNA in RNA structure buffer $\left(10 \mathrm{mM}\right.$ Tris $\left.\mathrm{pH} 7,0.1 \mathrm{M} \mathrm{KCl}, 10 \mathrm{mM} \mathrm{MgCl}_{2}\right)$ was heated to $95^{\circ} \mathrm{C}$ for $2 \mathrm{~min}$, stand on ice for $3 \mathrm{~min}$, then left at room temperature (RT) for $30 \mathrm{~min}$ to allow proper secondary structure formation. Samples were subjected to RNA pulldown using Pierce ${ }^{\mathrm{TM}}$ Magnetic RNA-Protein Pull-Down Kit (Thermo, 20164). Beads were eluted and boiled in $2 \times$ SDS protein loading buffer. The recovered proteins were subjected to gradient gel electrophoresis and mass spectrometry (MS) for protein identification or detected by western blot.

In vivo RNA pulldown. DNA probes for in vivo pulldown were ordered from Guangzhou RiboBio Co., LTD and labeled with biotin at 3' end. In vivo RNA pulldown was performed as previously described ${ }^{55}$. Briefly, $2 \times 10^{8}$ cells were washed with ice-cold PBS once, cross-linked with $265 \mathrm{~nm}$ UV light at $400 \mathrm{~mJ}$ energy in ice-cold PBS, treated with CSKT buffer supplemented with $1 \mathrm{mM} \mathrm{PMSF}$ and SUPERaseIn at $4{ }^{\circ} \mathrm{C}$ for $10 \mathrm{~min}$. After centrifuge for $10 \mathrm{~min}$ at $1200 \times g$, the pellet was resuspended in $3 \mathrm{~mL}$ of DNase I buffer $(50 \mathrm{mM}$ Tris $\mathrm{pH} 7.5,0.5 \%$ Nonidet-P $40,0.1 \%$ sodium lauroyl sarcosine, $1 \times$ protease inhibitors, SUPERaseIn, 600 U RNase free DNase I, $10 \mathrm{mM}$ vanadyl ribonucleoside complex) and incubated at $37^{\circ} \mathrm{C}$ to dissolve the pellet followed by a spin. The supernatant was pre-cleared by $50 \mu \mathrm{L}$ of M-280 streptavidin Dynabeads (Thermo, 00781251) at room temperature for $20 \mathrm{~min}$, and beads were discarded. Pre-cleared lysate, $100 \mathrm{pmol}$ of probes and $160 \mu \mathrm{L}$ beads were combined, incubated at room temperature for $20 \mathrm{~min}$, preheated to $65^{\circ} \mathrm{C}$ for $15 \mathrm{~min}$ and followed by slowly cooling to $37^{\circ} \mathrm{C}$ for $1 \mathrm{~h}$. The beads were washed $5 \mathrm{~min}$ for three times with wash buffer $1(50 \mathrm{mM}$ Tris, $\mathrm{pH} 7.5,0.3 \mathrm{M} \mathrm{LiCl}, 1 \%$ SDS, $0.5 \%$ Nonidet-P $40,1 \mathrm{mM}$ DTT, $1 \mathrm{mM}$ PMSF, $1 \times$ protease cocktail inhibitors (Roche)) at $37^{\circ} \mathrm{C}$, treated with $20 \mathrm{U}$ of DNase I in $300 \mu \mathrm{L}$ of DNase I buffer at $37^{\circ} \mathrm{C}$ for $10 \mathrm{~min}$. Beads were washed with wash buffer 1 twice at $37^{\circ} \mathrm{C}$ and buffer $2(1 \%$ SDS, $1 \mathrm{mM}$ DTT, $5 \mathrm{mM}$ EDTA, $150 \mathrm{mM} \mathrm{NaCl}$, $1 \mathrm{mM}$ PMSF) once. Proteins were eluted and boiled in $2 \times$ protein loading buffer at $100^{\circ} \mathrm{C}$ for $15 \mathrm{~min}$.

RNA immunoprecipitation (RIP). RIP was performed as previously described ${ }^{56,57}$ with minor modifications. Briefly, $2 \times 10^{7}$ cells were lysed in lysis buffer $(10 \mathrm{mM}$ HEPES pH 7.0, $100 \mathrm{mM} \mathrm{KCl,} 5 \mathrm{mM} \mathrm{MgCl}_{2}, 0.5 \% \mathrm{NP} 40,1 \mathrm{mM}$ DTT plus $0.5 \mathrm{U}$ SUPERaseIn (Thermo, AM2694) and proteinase inhibitors) at $-80^{\circ} \mathrm{C}$ overnight The lysates were centrifuged at $15,000 \times g$ for $15 \mathrm{~min}$ at $4{ }^{\circ} \mathrm{C}$. The supernatants were incubated with protein $\mathrm{A} / \mathrm{G}$ agrose (Abmart, A10001) precoated either with $2.5 \mu \mathrm{g}$ of normal rabbit IgG antibody (Sigma, I5006) or with $2.5 \mu \mathrm{g}$ of NCL antibody (Abcam, ab22758) for $4 \mathrm{~h}$ at $4{ }^{\circ} \mathrm{C}$. Then beads were washed 5 times with $1 \mathrm{~mL}$ of ice-cold NT2 buffer. To release RNA, beads were resuspended in $150 \mu \mathrm{L}$ of Proteinase K/NT2 buffer (1\% SDS, $2.4 \mu \mathrm{g} / \mu \mathrm{L}$ of Proteinase K) and incubated for $30 \mathrm{~min}$ at $55^{\circ} \mathrm{C}$. RNA was extracted with TRNzol Universal (Tiangen, DP424). The reverse transcription of the immunoprecipitated and input RNAs started from the same volume. Fold enrichment was calculated above sample-specific background as described $^{56}$.

DNA fiber assay. Cells were labeled with $50 \mu \mathrm{M}$ 5-iodo-2 ${ }^{\prime}$-deoxyuridine (IdU; Sigma, I7125) for $30 \mathrm{~min}$ and then incubated with $50 \mu \mathrm{M}$ 5-chloro- $2^{\prime}$-deoxyuridine (CldU; Sigma, C6891) for $30 \mathrm{~min}$, with or without treatment of hydroxyurea (HU, Selleck, S1896). DNA fibers were made as described ${ }^{7}$. Briefly, cells were harvested and suspended in PBS at a concentration of $10^{6} / \mathrm{mL}$. 2,500 cells were lyzed in $12 \mu \mathrm{L}$ of spreading buffer (0.5\% SDS, $50 \mathrm{mM}$ EDTA, $200 \mathrm{mM}$ Tris, pH 7.4) on one end of the glass slide. DNA fibers were spread along the slide by tilting the slides, fixed with freshly prepared methanol-acetic acid (3:1), and treated with $2.5 \mathrm{M}$ hydrochloric acid. For detection of labeled fibers, rat anti-BrdU/CIdU (BU1/75) monoclonal antibody (Novus, NB500-169) and mouse anti-IdU monoclonal antibody (BD, 347580) were used as the primary antibody. The secondary antibodies were AlexaFluor 488-conjugated goat anti-mouse IgG or Cy3-conjugated goat anti-rat IgG. Images were captured from Olympus confocal microscope. The length of labeled fibers were measured using the Image J software, and values were transformed into kilobases using the transform factor $1 \mu \mathrm{m}=2.59 \mathrm{~kb}$. At least 200 fibers were analyzed for stalled fork restart and nascent DNA degradation.

New replication origin firing analysis. Origin firing analysis was performed as described ${ }^{58}$. Briefly, cells were pulse-labeled with $50 \mu \mathrm{M}$ CIdU for $10 \mathrm{~min}$ without $\mathrm{HU}$ or with $0.1 \mathrm{mM} \mathrm{HU}$ for $20 \mathrm{~min}$. DNA fibers including at least four consecutive tracks were selected for measurement. At least 50 DNA fibers were analyzed.

Quantitative reverse transcription-polymerase chain reaction (qRT-PCR) Total RNA was isolated using Trizol (Tiangen) protocol. $1 \mu \mathrm{g}$ of total RNA was reverse transcribed using PrimeScript ${ }^{\mathrm{TM}}$ RT Reagent Kit with gDNA Eraser (Takara, RR037A), according to the manufacturer's protocol. qRT-PCR was performed on CFX96 ${ }^{\mathrm{TM}_{-}}$-Real Time Systerm (Biorad) using the TB Green ${ }^{\mathrm{ma}}$ Premix Ex $\mathrm{Taq}^{\mathrm{m}}$ II kit (Takara, RR820A). The sequences of the primers used were listed in Supplementary Table 1.

Discn copy number measurement. The cells were counted and lyzed in TRNzol Universal (Tiangen, DP424). The External RNA Controls Consortium (ERCC) RNA Spike-In Control Mixe 1 (Thermo, 4456740) was added into the cell lysate as control. RNAs were isolated for downstream qRT-PCR. ERCC-00042 was used to generate a standard curve for absolute quantification of ERCC-00042 abundance in recovered RNAs. The efficiency of RNA recovery was calculated as the ratio of recovered ERCC-00042 amount to initial ERCC-00042 amount. The copy numbers of Discn were determined by comparing $\mathrm{Ct}$ values of Discn to the $\mathrm{Ct}$ values of ERCC-00042, followed by calibration with the RNA recovery efficiency.

Laser micro-irradiation. Cells were plated on coverslips pre-coated with matrigel. A 405-nm laser of Olympus FV1000 confocal microscope was used for microirradiation. Cells were allowed to recover for $2 \mathrm{~h}$. To label S phase of cells, $50 \mu \mathrm{M}$ BrdU were added in the final $30 \mathrm{~min}$. Then cells were fixed in 4\% PFA and subjected for immunostaining. At least 50 cells per experiment were analyzed. Three independent experiments were performed.

DNA repair assay using HR-RFP and NHEJ-GFP reporter cells. Mouse ESC lines containing NHEJ reporter and HR reporter, and the I-SceI expression plasmid, pcDNA3 $\beta$-I-SceI were kindly provided by professor An-yong Xie, School of Medicine, Zhejiang University. mESC reporters were transfected with pcDNA3 $\beta-\mathrm{I}$ SceI and GFP or RFP expression plasmid using Neon transfection system. At three days post-transfection, cells were analyzed for $\mathrm{RFP}^{+}$or $\mathrm{GFP}^{+}$frequencies using a FACS LSRFortessa flow cytometer (BD), BD FACSDiva software (Version 8.0.2), and Flowjo software (Version 7.6). The gating strategies for HR-RFP ${ }^{+}$cells and NHEJ-GFP ${ }^{+}$cells were presented in Supplementary Fig. 8.

Immunoprecipitation and western blotting. Cells were lysed in RIPA buffer (Beyotime, P0013J) supplemented with $1 \times$ proteinase inhibitor (Beyotime, P1006) Immunoprecipitation with $2.5 \mu \mathrm{g}$ of RPA32 antibody or $2.5 \mu \mathrm{g}$ of isotype IgG (Sigma, I5006) per sample was performed using Protein G Dynabeads (Thermo, 88847) according to the manufacturers' protocol.

For western blot, total protein was extracted using RIPA buffer (Beyotime, P0013J). Proteins were subjected to 4\%-12\% SDS-PAGE gels for separation and transferred to polyvinylidene fluoride (PVDF) membrane (Roche, 03010040001) The membrane was blocked with $5 \%$ non-fat milk at room temperature for $2 \mathrm{~h}$ and then incubated with primary antibodies at $4{ }^{\circ} \mathrm{C}$ overnight. After incubation with anti-mouse or rabbit HRP-conjugated secondary antibodies for $1 \mathrm{~h}$ at room temperature, the bands were revealed using ECL reagent (Beyotime, P0018FS) Antibodies used were listed in Supplementary Table 2.

Mouse behavioral tests. All behavioral tests were performed using two months old male mice from littermates. All experiments and data analyses were performed in a blinded manner. The behavioral studies including open-field test, light-dark shuttle box test, Morris water maze test and self-grooming test were performed as previously reported ${ }^{29}$. Briefly, Morris water maze test was carried out in a tank with $120-\mathrm{cm}$ diameter and $50-\mathrm{cm}$ depth. The tank was equipped with a $10-\mathrm{cm}$ diameter platform submerged $1 \mathrm{~cm}$ under the water surface masked using white beads. Mice were trained to find the platform within $60 \mathrm{~s}$ and stay on the platform for $15 \mathrm{~s}$ Training was terminated once one of the groups succeeded in landing on the platform within $10 \mathrm{~s}$. At 4 and $72 \mathrm{~h}$ following the last training, the platform was removed and mice were tested to locate the platform within $60 \mathrm{~s}$ in the pool. Mice were video-tracked and their trace was analyzed using the SMART 3.0 software (Panlab Harvard, MA, USA). 
Open-field test was performed in an open-top chamber ( 40 inches by 40 inches by 40 inches). Each mouse was placed in a chamber for $1 \mathrm{~h}$. The activities of mice in the box, including the time spent in the center area and entries to center area, were vedieo-tracked and analyzed by the SMART 3.0 software (Panlab Harvard, MA, USA).

Light-dark box test was performed in an instrument composed of a $18 \mathrm{~cm}$ by $27 \mathrm{~cm}$ black box and a $27 \mathrm{~cm}$ by $27 \mathrm{~cm}$ light box. Mice were initially placed into the dark box and allowed to shuttle to the light box for $30 \mathrm{~min}$. The time spent in light box was analyzed.

For self-grooming test, mice were placed in a new cage filled with fresh bedding. Total time spent on grooming and the numbers of grooming bouts were recorded for $10 \mathrm{~min}$.

For rotarod test, the rotation speed of rod was 20 revolutions per minute (rpm) during each trial that lasted for $3 \mathrm{~min}$. Each mouse was tested for three trials with an interval of $30 \mathrm{~min}$. The time spent on the rotating rod was used for evaluation of the motor coordination and balance ${ }^{59}$.

Statistical analyses. Statistical analyses were performed by two-tailed Student's $t$ test, two-way ANOVA with Bonferroni post using GraphPad Prism (Version 8.0.1). $P<0.05$ was considered significant. Statistical details of the experiments were described in the figure legend or in the methods section. $P$ values were labeled on the panels. All experiments were performed at least three times.

Reporting summary. Further information on research design is available in the Nature Research Reporting Summary linked to this article.

\section{Data availability}

The data that support this study are available from the corresponding author upon reasonable request. The sequence of Discn has been deposited in GenBank under accession number MZ269527. RNA-seq data are available in Gene Expression Omnibus database under accession number GSE161998. The mouse reference genome ( $\mathrm{mm} 9$ and mm10) and annotations can be downloaded through the Illumina's iGenomes project (http://ccb.jhu.edu/software/tophat/igenomes.shtml). Non-coding transcript annotation file can be downloaded from the NONCODE database (http://www.noncode.org/). For each figure, raw data are provided in the source data files. Source data are provided with this paper.

\section{Code availability}

The "gplots" code for data visualization ( $\mathrm{R}$ version 3.6.1) are available from the corresponding author on reasonable request.

Received: 3 February 2021; Accepted: 31 August 2021; Published online: 22 September 2021

\section{References}

1. Vitale, I., Manic, G., De Maria, R., Kroemer, G. \& Galluzzi, L. DNA damage in stem cells. Mol. Cell 66, 306-319 (2017).

2. Zalzman, M. et al. Zscan4 regulates telomere elongation and genomic stability in ES cells. Nature 464, 858-863 (2010).

3. Ruis, P. et al. TRF2-independent chromosome end protection during pluripotency. Nature 589, 103-109 (2021).

4. Markiewicz-Potoczny, M. et al. TRF2-mediated telomere protection is dispensable in pluripotent stem cells. Nature 589, 110-115 (2021).

5. Xiong, J. et al. Stemness factor Sall4 is required for DNA damage response in embryonic stem cells. J. Cell Biol. 208, 513-520 (2015).

6. Zhao, B. et al. Filia is an ESC-specific regulator of DNA damage response and safeguards genomic stability. Cell Stem Cell 16, 684-698 (2015).

7. Zhao, B. et al. Mouse embryonic stem cells have increased capacity for replication fork restart driven by the specific Filia-Floped protein complex. Cell Res 28, 69-89 (2018).

8. Caldwell, C. C. \& Spies, M. Dynamic elements of replication protein A at the crossroads of DNA replication, recombination, and repair. Crit. Rev. Biochem. Mol. Biol. 55, 482-507 (2020).

9. Li, S., Dong, Z., Yang, S., Feng, J. \& Li, Q. Chaperoning RPA during DNA metabolism. Curr. Genet. 65, 857-864 (2019).

10. Maréchal, A. \& Zou, L. RPA-coated single-stranded DNA as a platform for post-translational modifications in the DNA damage response. Cell Res $\mathbf{2 5}$, 9-23 (2015).

11. Mujoo, K., Butler, E. B., Pandita, R. K., Hunt, C. R. \& Pandita, T. K. Pluripotent stem cells and DNA damage response to ionizing radiations. Radiat. Res. 186, 17-26 (2016).

12. Kopp, F. \& Mendell, J. T. Functional classification and experimental dissection of long noncoding RNAs. Cell 172, 393-407 (2018).
13. Nagy, Z. \& Soutoglou, E. DNA repair: easy to visualize, difficult to elucidate Trends Cell Biol. 19, 617-629 (2009).

14. Swain, U. \& Subba Rao K. Study of DNA damage via the comet assay and base excision repair activities in rat brain neurons and astrocytes during aging. Mech. Ageing Dev. 132, 374-381 (2011).

15. Zeman, M. K. \& Cimprich, K. A. Causes and consequences of replication stress. Nat. Cell Biol. 16, 2-9 (2014).

16. Sørensen, C. S. et al. The cell-cycle checkpoint kinase Chk1 is required for mammalian homologous recombination repair. Nat. Cell Biol. 7, 195-201 (2005).

17. Técher, H. et al. Replication dynamics: biases and robustness of DNA fiber analysis. J. Mol. Biol. 425, 4845-4855 (2013).

18. Seluanov, A., Mao, Z. \& Gorbunova, V. Analysis of DNA double-strand break (DSB) repair in mammalian cells. J. Vis. Exp. 8, 2002 (2010).

19. Iyama, T. \& Wilson, D. M. 3rd DNA repair mechanisms in dividing and nondividing cells. DNA Repair (Amst.) 12, 620-636 (2013).

20. Abdelmohsen, K. et al. Enhanced translation by Nucleolin via G-rich elements in coding and non-coding regions of target mRNAs. Nucleic Acids Res 39, 8513-8530 (2011)

21. Chang, T. H. et al. An enhanced computational platform for investigating the roles of regulatory RNA and for identifying functional RNA motifs. BMC Bioinforma. 14(Suppl 2), S4 (2013).

22. Kim, K. et al. Novel checkpoint response to genotoxic stress mediated by nucleolin-replication protein a complex formation. Mol. Cell Biol. 25, 2463-2474 (2005)

23. Daniely, Y. \& Borowiec, J. A. Formation of a complex between nucleolin and replication protein A after cell stress prevents initiation of DNA replication. J. Cell Biol. 149, 799-810 (2000).

24. Wu, Y., Lu, J. \& Kang, T. Human single-stranded DNA binding proteins: guardians of genome stability. Acta Biochim. Biophys. Sin. (Shanghai) 48, 671-677 (2016).

25. Toledo, L. I. et al. ATR prohibits replication catastrophe by preventing global exhaustion of RPA. Cell 155, 1088-1103 (2013).

26. Toledo, L., Neelsen, K. J. \& Lukas, J. Replication catastrophe: when a checkpoint fails because of exhaustion. Mol. Cell 66, 735-749 (2017).

27. Elguindy, M. M. \& Mendell, J. T. NORAD-induced Pumilio phase separation is required for genome stability. Nature 595, 303-308 (2021).

28. Vasquez, V. et al. Chromatin-bound oxidized $\alpha$-Synuclein causes strand breaks in neuronal genomes in in vitro models of Parkinson's Disease. $J$. Alzheimers Dis. 60, S133-S150 (2017).

29. Li, J. et al. Genome integrity and neurogenesis of postnatal hippocampal neural stem/progenitor cells require a unique regulator Filia. Sci. Adv. 6 eaba0682 (2020).

30. Fragola, G. et al. Deletion of Topoisomerase 1 in excitatory neurons causes genomic instability and early onset neurodegeneration. Nat. Commun. 11, 1962 (2020).

31. Briard, B., Place, D. E. \& Kanneganti, T. D. DNA sensing in the innate immune response. Physiol. (Bethesda) 35, 112-124 (2020).

32. Reisländer, T., Groelly, F. J. \& Tarsounas, M. DNA damage and cancer immunotherapy: a STING in the tale. Mol. Cell 80, 21-28 (2020).

33. Dunphy, G. et al. Non-canonical activation of the DNA sensing adaptor STING by ATM and IFI16 mediates NF- $\kappa$ B signaling after nuclear DNA damage. Mol. Cell 71, 745-760 (2018)

34. Fernandes-Alnemri, T., Yu, J. W., Datta, P., Wu, J. \& Alnemri, E. S. AIM2 activates the inflammasome and cell death in response to cytoplasmic DNA. Nature 458, 509-513 (2009)

35. McNairn, A. J., Chuang, C. H., Bloom, J. C., Wallace, M. D. \& Schimenti, J. C. Female-biased embryonic death from inflammation induced by genomic instability. Nature 567, 105-108 (2019).

36. Dumitru, R. et al. Human embryonic stem cells have constitutively active Bax at the Golgi and are primed to undergo rapid apoptosis. Mol. Cell 46, 573-583 (2012).

37. Heyer, B. S., MacAuley, A., Behrendtsen, O. \& Werb, Z. Hypersensitivity to DNA damage leads to increased apoptosis during early mouse development. Genes Dev. 14, 2072-2084 (2000)

38. Wei, P. C. et al. Long neural genes harbor recurrent DNA break clusters in neural stem/progenitor cells. Cell 164, 644-655 (2016)

39. Wei, P. C. et al. Three classes of recurrent DNA break clusters in brain progenitors identified by 3D proximity-based break joining assay. Proc. Natl Acad. Sci. USA 115, 1919-1924 (2018).

40. Motwani, M., Pesiridis, S. \& Fitzgerald, K. A. DNA sensing by the cGASSTING pathway in health and disease. Nat. Rev. Genet. 20, 657-674 (2019).

41. Estes, M. L. \& McAllister, A. K. Maternal immune activation: Implications for neuropsychiatric disorders. Science 353, 772-777 (2016).

42. Belenguer, G. et al. Adult neural stem cells are alerted by systemic inflammation through TNF- $\alpha$ receptor signaling. Cell Stem Cell 28, 285-299 (2021).

43. Middelkamp S. et al. Sperm DNA damage causes genomic instability in early embryonic development. Sci. Adv. 6, eaaz7602 (2020). 
44. Tan, Y. S. \& Lei, Y. L. Generation and culture of mouse embryonic fibroblasts. Methods Mol. Biol. 1960, 85-91 (2019).

45. Trapnell, C. et al. Transcript assembly and quantification by RNA-Seq reveals unannotated transcripts and isoform switching during cell differentiation. Nat. Biotechnol. 28, 511-515 (2010).

46. Sun, L. et al. Utilizing sequence intrinsic composition to classify proteincoding and long non-coding transcripts. Nucleic Acids Res 41, e166 (2013).

47. Wang, L. K., Feng, Z. X., Wang, X., Wang, X. W. \& Zhang, X. G. DEGseq: an $\mathrm{R}$ package for identifying differentially expressed genes from RNA-seq data. Bioinformatics 26, 136-138 (2010).

48. Ashburner, M. et al. Gene Ontology: tool for the unification of biology. Nat. Genet. 25, 25-29 (2000).

49. Carbon, S. et al. The Gene Ontology resource: 20 years and still Going strong. Nucleic Acids Res 47, D330-D338 (2019).

50. Picelli, S. et al. Full-length RNA-seq from single cells using Smart-seq2. Nat. Protoc. 9, 171-181 (2014).

51. Stults, D. M., Killen, M. W., Marco-Casanova, P. \& Pierce, A. J. The sister chromatid exchange (SCE) assay. Methods Mol. Biol. 2102, 441-457 (2020).

52. Hacot S. et al. Isolation of nucleoli. Curr. Protoc. Cell Biol. Chapter 3, Unit3.36 (2010).

53. Atianand, M. K. et al. A long noncoding RNA lincRNA-EPS acts as a transcriptional brake to restrain inflammation. Cell 165, 1672-1685 (2016).

54. Wang, P. et al. The STAT3-binding long noncoding RNA lnc-DC controls human dendritic cell differentiation. Science 344, 310-313 (2014).

55. Chu, H. P. et al. TERRA RNA antagonizes ATRX and protects telomeres. Cell 170, 86-101 (2017).

56. Gagliardi, M. \& Matarazzo, M. R. RIP: RNA immunoprecipitation. Methods Mol. Biol. 1480, 73-86 (2016).

57. Keene, J. D., Komisarow, J. M. \& Friedersdorf, M. B. RIP-Chip: the isolation and identification of mRNAs, microRNAs and protein components of ribonucleoprotein complexes from cell extracts. Nat. Protoc. 1, 302-307 (2006).

58. Ge, X. Q. et al. Embryonic stem cells license a high level of dormant origins to protect the genome against replication stress. Stem Cell Rep. 5, 185-194 (2015).

59. Niu, J., Cao, Y. Q. \& Ji, Y. J. Resveratrol, a SIRT1 activator, ameliorates MK801-induced cognitive and motor impairments in a neonatal rat model of schizophrenia. Front. Psychiatry 11, 716 (2020).

\section{Acknowledgements}

We thank Professor An-yong Xie in Zhejiang University School of Medicine for providing the HR-RFP and NHEJ-GFP reporter mouse ESCs, Professor Baowei Jiao in Kunming Insitute of Zoology, CAS for providing the cDNA of mouse breast stem cell, Prof. Bo Zhao in Kunming Insitute of Zoology, CAS for critical reading and suggestions on the manuscript. This work was supported by the National Natural Science Foundation of China to P.Z. (31930027), National Key Research and Development Program of China, Stem Cell and Translational Research (2016YFA0100300), and Young Scientists Fund of National Natural Science Foundation of China to L.W. (31801223).

\section{Author contributions}

L.W., JZ.L., and WD. Z. performed the experiments, analyzed the data, and participated in the experimental design and manuscript preparation. H.Z. and J.G. performed mass spectrometry analysis. P.Z. supervised the study and wrote the manuscript.

\section{Competing interests}

The authors declare no competing interests.

\section{Additional information}

Supplementary information The online version contains supplementary material available at https://doi.org/10.1038/s41467-021-25827-6.

Correspondence and requests for materials should be addressed to Ping Zheng.

Peer review information Nature Communications thanks the anonymous reviewer(s) for their contribution to the peer review of this work.

Reprints and permission information is available at http://www.nature.com/reprints

Publisher's note Springer Nature remains neutral with regard to jurisdictional claims in published maps and institutional affiliations.

(c) (i) Open Access This article is licensed under a Creative Commons Attribution 4.0 International License, which permits use, sharing, adaptation, distribution and reproduction in any medium or format, as long as you give appropriate credit to the original author(s) and the source, provide a link to the Creative Commons license, and indicate if changes were made. The images or other third party material in this article are included in the article's Creative Commons license, unless indicated otherwise in a credit line to the material. If material is not included in the article's Creative Commons license and your intended use is not permitted by statutory regulation or exceeds the permitted use, you will need to obtain permission directly from the copyright holder. To view a copy of this license, visit http://creativecommons.org/ licenses/by/4.0/.

(c) The Author(s) 2021 